\title{
9 Zwangsstörungen und Kultur
}

\author{
David Holzer
}

Die ersten Berichte über das Leid von Menschen, welche von zwanghaften Phänomenen verfolgt wurden, finden sich bereits im überlieferten Schrifttum der Babylonier und des antiken Griechenland (Reynolds u. Kinnier Wilson 2012; Zaudig 2011; Greenberg u. Huppert 2010). In vorwissenschaftlichen Darstellungen des psychischen Zwangs, wurde vor allem dessen Verbindung mit religiösen Themen hervorgehoben. So wird etwa in der wichtigsten Sammlung religionsgesetzlicher Überlieferungen des Judentums, der Mischna, vor einem Übermaß an Besorgnis gewarnt, das bei der Reinigung des Hauses vor dem Pessachfest auftreten kann (Greenberg u. Huppert 2010). Der Babylonische Talmud wiederum berichtet von Gläubigen, die ihre Gebete unaufhörlich wiederholen müssen, da sie befürchten, bei deren Ausführung nicht ausreichend aufmerksam gewesen zu sein (Reynolds u. Kinnier Wilson 2012).

Während des Mittelalters wurden religiöse Zwänge in Europa den Besessenheitszuständen zugerechnet und vorwiegend durch das Wirken des Teufels erklärt. Der Begriff „obsessiones“ (lat. obsidere: belagern, besetzen) bezeichnete dabei auch den inneren Drang, in der Kirche gotteslästerliche Dinge laut auszusprechen, also „mit der Zunge des Teufels“ zu sprechen (Greenberg u. Huppert 2010). Im 15. und 16. Jahrhundert wurde in der christlichen Moraltheologie der Begriff „Skrupel“ eingeführt um damit ein Übermaß an Gewissenhaftigkeit und Zweifel bezüglich religiöser Inhalte zu bezeichnen. In Anlehnung an das lateinische „scrupulum“ fand dabei dessen Bedeutung als „kleines spitzes Steinchen“ sinnbildhafte Verwendung, da das moralische Gewissen den Betroffenen wie ein „spitzer Stein im Schuhwerk“ unablässig quälte.

Da im Christentum des Mittelalters Vorstellungen als ebenso schwerwiegend wie Taten angesehen wurden, galt auch das bewusste Verweilen bei bestimmten Gedanken als sündhaft und musste gebeichtet werden. Skrupelhafte Christen neigten deshalb zu übermäßig häufiger Beichte und begehrten immer wieder die Lossprechung von ihren Sünden, da sie von wiederkehrenden Zweifeln 
an der Vollständigkeit und Aufrichtigkeit ihrer Buße gequält wurden (Morschitzky 2009). Des Weiteren konnte auch die Aufmerksamkeit beim Gebet, die Einhaltung von Gelübden, die Beachtung der Ordensregeln oder die Möglichkeit von Ehehindernissen Gegenstand von Skrupeln werden (Morschitzky 2009) So sollen auch namhafte Persönlichkeiten der Religionsgeschichte, wie der Reformator Martin Luther (1483-1546), der Gründer des Jesuitenordens Ignatius von Loyola (14911556) oder der Baptistenprediger John Bunyan (1628-1688), unter dieser Übergewissenhaftigkeit gelitten haben. Während zwanghafte Verhaltensweisen somit bis zur Neuzeit vorwiegend als religiöse Probleme betrachtet und durch das Wirken des Teufels erklärt wurden, werden sie seit dem 19. Jahrhundert den psychopathologischen Phänomenen zugeordnet.

\subsection{Historischer Abriss}

Die Grundzüge einer Zwangsstörung wurden erstmals von Jean Etienne Dominique Esquirol (1782-1840) in seiner 1839 veröffentlichten Kasuistik der Mademoiselle F. beschrieben (Esquriol 1839). Der Schüler Philippe Pinels (17451826) hob dabei insbesondere die erhaltene Intelligenz sowie die Einsicht der Patientin in die Absurdität ihrer zwanghaften Ängste hervor, weshalb er das beschriebene Zustandsbild auch als Ausdruck einer Störung des Denkens auffasste (Zaudig 2011a; Loewenfeld 1904; Wille 1882). 1866 wurde von Bénédict-Augustin Morel (1809-1873) jedoch eine Krankheitseinheit definiert, die er als „Délire émotif“ bezeichnete und welcher er die zwanghaften Erscheinungen zurechnete. Mit Einführung dieser Kategorie wurde die Zwangsstörung in Frankreich fortan als Störung des Gemüts betrachtet (Berrios 1986; Loewenfeld 1904).

Der deutsche Begriff „Zwang“ wurde von dem Psychiater und Rechtsmediziner Richard Freiherr von Krafft-Ebing (1840-1902) in die deutsche Fachsprache eingeführt. In seiner 1867 veröffentlichen Schrift „Beiträge zur Erkennung und richtigen forensischen Beurteilung krankhafter Gemütszustände für Ärzte, Richter und Verteidiger“ beschrieb er negative Vorstellungen, die sich dem Gemütsdepressiven unwillkürlich aufdrängen, das Bewusstsein belagern und so den freien Fluss der Assoziationen blockieren (Krafft-Ebing 1867). KrafftEbing bezeichnete diese Gedanken als „Zwangsvorstellungen“ und führte dazu aus, dass wo der freie Wille, die schrankenlose Assoziation der Vorstellung gehemmt ist, auch das Wollen, wie es der Begriff der Zurechnungsfähigkeit voraussetzt, nicht mehr bestehen kann, wobei es schließlich zu einem unbeherrschten Drang, einem Zwangswollen, wird. (Krafft-Ebing 1867). In einer weiteren, 1870 veröffentlichten, Arbeit besprach Krafft-Ebing erneut den Einfluss der Zwangsvorstellungen auf die Selbstbestimmungsfähigkeit, wobei er auch hier ausschließlich psychische Phänomene in Betracht zog, die von einem depressiven Affekt getragen wurden (Loewenfeld 1904; Wille 1882).

Am 23. März 1868 berichtete Wilhelm Griesinger (1817-1868) vor der Berliner Medizinisch-Psychologischen Gesellschaft „Über einen wenig bekannten psychopathischen Zustand“, die krankhafte „Grübelsucht“. Die Ausführungen Griesingers stützten sich dabei auf die Beobachtungen von drei Patienten, die, wie er ausdrücklich betonte, nicht in einer „Irrenanstalt“ aufgenommen waren. 
Die geschilderten Zustände sollten dabei nach seiner Ansicht allein in dem von Falret beschriebenen Leiden, der „Maladie du doute“ (Zweifelsucht), eine gewisse Analogie finden (Griesinger 1868). 1875 definierte der französische Psychiater Henri Legrand du Saulle (1830-1886) in seiner Arbeit „La folie du doute (avec délire du toucher)“ erstmals das Zwangssyndrom als eigenständiges Krankheitsbild (Loewenfeld 1904; Berrios 1989).

Ein Vortrag, den Carl Westphal (1833-1890) im Jahre 1877 vor der Berliner Medizinisch-Psychologischen Gesellschaft hielt, sollte für die weitere Konzeptentwicklung der Zwangsstörung maßgebend sein, da in diesem eine bis heute weitgehend gültige Begriffsbestimmung des pathologischen Zwangs gegeben wurde. Westphal berichtete nämlich von Zwangsvorstellungen, „welche, bei übrigens intakter Intelligenz und ohne durch einen Gefühls- oder affektartigen Zustand bedingt zu sein, gegen und wider den Willen des betreffenden Menschen in den Vordergrund des Bewusstseins treten, sich nicht verscheuchen lassen, den normalen Ablauf der Vorstellungen hindern und durchkreuzen, welche der Befallene stets als abnorm, ihm fremdartige anerkennt, und denen er mit seinem gesunden Bewusstsein gegenübersteht" (Westphal 1878). Vor dem Kollegium der königlichen Gesellschaft der Ärzte in Budapest schlug Julius Donath (1870-1950) schließlich den Begriff „Anancasmus“ als Bezeichnung für das neue Konzept der idiopathischen Zwangserkrankung (Thomson 1895) vor, der noch heute als Synonym für die zwanghafte Verhaltensweisen in Verwendung ist (Donath 1896; Berrios 1996).

Nach der Ansicht vieler deutschsprachiger Psychiater jener Zeit handelte es sich bei den Zwangszuständen um eine Störung der intellektuellen Funktion und nicht, wie es von der französischen Psychiatrie seit Morel behauptet wurde, um eine Beeinträchtigung des emotionalen Seelenlebens (Berrios 1996). Zwangshandlungen wurden dabei jedoch übereinstimmend als sekundär oder zumindest in Abhängigkeit zu den Zwangsgedanken stehend verstanden. Nach Berrios (1996) führte diese Ambiguität dazu, dass in Großbritannien Zwangsphänomene als „obsessions“ bezeichnet wurden, während sie in den Vereinigten Staaten „compulsions“ genannt wurden. Da es im Englischen auch keine passende Übersetzung für den deutschen Begriff Zwang gibt, setzte sich als internationaler Kompromiss die Doppelbezeichnung „obsessivecompulsive“ durch (Zaudig 2011a; Berrios 1996).

Das Konzept des psychischen Zwangs wurde in der Folge von namhaften Seelenärzten wie Pierre Janet, Sigmund Freud oder Kurt Schneider bearbeitet, wobei sich in der Allgemeinen Psychopathologie von Karl Jaspers (1913) eine plastische Beschreibung der Zwangsstörung findet.

„Der Zwangskranke wird verfolgt von Vorstellungen, die ihm nicht nur als fremd, sondern unsinnig erscheinen, und denen er doch folgen muss, als ob sie wahr seien. Tut er es nicht, so befällt ihn grenzenlose Angst. Der Kranke zum Beispiel muss etwas tun, sonst stirbt eine Person oder es geschieht ein Unheil. Es ist, als ob sein Tun und Denken magisch das Geschehen verhinderte oder bewirke. Er baut seine Gedanken zu einem System von Bedeutungen, seine Handlungen zu einem System von Zeremonien und 
Riten aus. Aber jede Ausführung hinterlässt den Zweifel, ob er es auch richtig, auch vollständig macht. Der Zweifel zwingt ihn, von vorne anzufangen." (Jaspers 1913)

\subsection{Aspekte des Zwangsbegriffs}

Die deutsche Bezeichnung „Zwang“ ist ein Verbalabstraktum und findet ihre wortgeschichtlichen Wurzeln im althochdeutschen Begriff thwanga, welcher das Bedeutungsfeld des „Zusammenpressens“ und „Drückens“ umfasste. Im Allgemeinen zumeist als „Einschränkung der Entscheidungs- und Handlungsfreiheit" definiert kann Zwang in Anlehnung an seine etymologische Herkunft als imperativer Drang, eine Handlung gegen den eigenen Willen ausführen oder unterlassen zu müssen, aufgefasst werden. Der aversive Imperativ wird dabei durch bestimmende Einflüsse hervorgerufen, die als übermächtig oder bedrohlich erfahren werden. So mag sich ein Individuum etwa durch die Androhung von Gewalt durch Dritte oder die Unabänderlichkeit situativer Gegebenheiten zu bestimmten Handlungen gedrängt fühlen, welche nicht Ausdruck eines subjektiven Wunsches sind und daher als fremdbestimmt erfahren werden.

Von einem Zwang durch äußere Einflüsse muss das psychopathologische Phänomen des inneren Zwangs abgegrenzt werden, das durch seelische Vorgänge hervorgerufen wird. Es ist dabei von entscheidender Bedeutung, dass sowohl der Begriff des äußeren als auch jener des inneren Zwangs grundsätzlich nicht auf ein objektives Phänomen verweisen, welches unabhängig von einer selbstreflexiven Betrachtung existiert, sondern beide eine subjektive Erfahrung repräsentieren, welche auf den Wünschen und Bewertungen des Betroffenen basiert (Bieri 2001). Das Zwangserleben an sich ist folglich untrennbar mit dem Selbstbewusstsein verbunden und nur auf dem Boden eines willkürlich geleiteten Seelenlebens möglich (Schneider 1946).

Karl Jaspers (1913) ordnete den psychischen Zwang deshalb unter die verstehbaren reflexiven Phänomene ein und sah in der widerstrebenden Stellungnahme des Subjekts zu sich selbst ein wesentliches Merkmal desselben. Bezüglich des psychopathologischen Zwangsbegriffs unterschied er zwei Extensionsformen, wobei er die Auffassung im weiteren Sinne, jener im engeren Sinne gegenüberstellte. Im weiteren Begriffsumfang wird nach Jaspers der Zwangscharakter eines seelischen Vorgangs allein durch das formale Merkmal des Auftretens charakterisiert, während er im engeren Sinne zusätzlich durch das inhaltliche Kriterium der Fremdheit gekennzeichnet ist (Jaspers 1913, Bürgy 2005).

Der Zwangsbegriff im weiteren Sinne verweist auf seelische Inhalte, welche mit Beharrlichkeit im Bewusstsein auftreten und wiederkehren, wodurch sie $z u$ einer Störung der intendierten Aktivität führen. In selbstreflexiver Stellungnahme wehrt sich das Subjekt gegen die störenden Einflüsse, ist gleichsam jedoch nicht imstande, sich von diesen abzuwenden. Die Dominanz der seelischen Inhalte gegenüber dem willentlichen Widerstreben verleiht diesen 
den bedrängenden Charakter des Zwanges und ruft das Gefühl hervor, ihrer überwältigenden Natur machtlos ausgeliefert zu sein (Stompe 2011). Nach Leopold Loewenfeld (1904) sind psychische Zwangserscheinungen in diesem Sinne seelische Elemente, welche der normalen Verdrängbarkeit durch Willenseinflüsse ermangeln und aufgrund ihrer Immobilität den normalen Verlauf der psychischen Prozesse stören.

Die engere Auffassung des Zwangsbegriffs zeichnet sich zusätzlich durch das Kriterium der inhaltlichen Fremdheit im Sinne einer ablehnenden Stellungnahme aus. Das Subjekt bewertet dabei den Inhalt der aufdrängenden Ideen als grundlos, unsinnig und relativ unverständlich (Bürgy 2005). Trotz der Gewissheit ihrer Urheberschaft, vermag sich der Betroffene nicht mit den Inhalten zu identifizieren, da sie dem Selbstbild oder der eigenen Wertewelt widersprechen. Zwang im engeren Sinne bezieht sich somit auf psychische Phänomene, gegen deren Dasein sich der Erlebende wehrt und deren Inhalt als ichfremd erlebt wird (Jaspers 1913).

Die Begriffsanalyse von Jaspers führt nun in aller Klarheit vor Augen, dass in einer weiteren Auffassung, aufgrund ihrer geringen Intension, zahlreichen psychischen Erscheinungen ein Zwangscharakter zugesprochen werden kann. Mit dem umfangreicheren Begriffsinhalt des engeren Verständnisses ist es hingegen möglich eine umschriebene Gruppe seelischer Symptome zu erfassen, welche nosologische Spezifität besitzen und für die Zwangsstörung charakteristisch sind. Die Besinnung auf die Struktur des Zwangs im engeren Sinne erlaubt daher eine klare Abgrenzung gegenüber anderen Phänomenen, welche einen zwangsähnlichen Charakter besitzen (Bürgy 2005).

In Zusammenschau der angeführten Aspekte kann schließlich festgehalten werden, dass der psychische Zwang einem imperativen Erlebnis entspricht, das durch die Unbeeinflussbarkeit des eigenen Willens und seiner Fremdheit im Sinne einer Ablehnung gekennzeichnet ist (Bieri 2001; Scharfetter 1976). Das unkontrollierbare Auftreten und die inhaltliche Fremdartigkeit der auslösenden Ideen nähren dabei die bedrängende Kraft des Zwangs, der für das Subjekt wie ein Fremdkörper im Inneren wuchert und dieses in seiner gesamten Existenz bedroht. Das angstbesetzte Zwangserleben kann schließlich zu bestimmten Handlungen führen, die von einem Subjekt in der Absicht vollzogen werden, der existenziellen Bedrohung auszuweichen (Bieri 2001).

In seiner 1936 veröffentlichten Monographie über die Psychologie der Zwangsvorgänge definierte der Schweizer Psychiater Hans Binder in entsprechender Weise, dass das Erlebnis eines psychischen Zwanges aus dem Zusammenwirken zweier Komponenten resultiere, welche er „Störungspsychismus“ und „Abwehrpsychismus“ bezeichnete. Das Zwangserleben würde demnach dann auftreten, wenn das Ich durch einen inneren Willensakt vergeblich versucht, eine Störung durch seelische Erscheinungen innerlich radikal zu unterdrücken, die als fremdartig und unsinnig erlebt werden, an dessen Zustandekommen sich aber das Ich trotzdem als aktiv beteiligt spürt. Diesem primären Störungs- 
vorgang (Störungspsychismus), welcher durch unkontrollierbar auftretende und fremdartige Bewusstseinsinhalte hervorgerufen wird, folgt eine zwangsmäßige Reaktion, der „Abwehrpsychismus“, welcher als Versuch des Subjekts angesehen werden kann, die Kohärenz und Autonomie der eigenen Person zu stabilisieren (Binder 1936; Gebsattel 1938).

Der deutsche Humanmediziner und Psychiater Viktor Emil von Gebsattel (18831976) sah in der „anankastischen Phobie“ die Grundlage der Störungsseite des Zwangssyndroms, welche als „phobische Besessenheit“, „phobische Ekelstimmung“ oder "Gewissensangst“ das Dasein des Zwangskranken bestimmt. Durch die Allgegenwart des „Unreinen“, „Ekelerregenden“ und „Verwerflichen“ erscheint der Betroffene „entweltlicht“ und „gespalten“, stets zurückgeworfen auf seine „Besessenheit“, die in unaufhörlich verfolgt und quält. Das Widerfahrnis des „Störungspsychismus“ (Binder 1936) stelle nach Gebsattel ferner ein Leiden dar, welches umgehend eine Handlung, eine Flucht oder Gegenwehr, den „Abwehrpsychismus“, erzwingt, wobei sich beide Vorgänge wie Zauber und Gegenzauber in einem unendlichen Kampf befinden. Gesiegt hat nämlich die „phobische Besessenheit“ jedes Mal dann, wenn die Maßnahmen, die ihre Abwehr erzwingen wollen, die Zwangshandlungen (Kompulsionen), selbst die Konkretisierung des latent phobischen Zustandes, die Zwangsgedanken (Obsessionen) herbeiführen und damit die neutralisierende Zauberhandlung zur Ohnmacht verdammen (Gebsattel 1939).

Die strukturdynamische Beziehung zwischen dem primären „Störungspsychismus“, dem sekundären „Abwehrpsychismus“ sowie der "phobischen Besessenheit" soll in Abbildung 35 zusammen mit dem modernen Modell der Zwangsstörung (Obsessionen, Kompulsionen und Disstress/Angst) dargestellt werden.

Das Phänomen des psychischen Zwangs wurde von Kurt Schneider (1946) in seiner Klinischen Psychopathologie äußerst stringent definiert:

„Zwang ist, wenn jemand Bewusstseinsinhalte nicht loswerden kann, obschon er sie gleichzeitig als inhaltlich unsinnig oder wenigstens als ohne angemessenen Grund beherrschend und beharrend beurteilt. Will man ganz kritisch sein, so-sage man, von innen kommende' Bewusstseinsinhalte, um die Zwangserlebnisse begrifflich gegen die von außen gemachten Erlebnisse Schizophrener abzudichten." (Schneider 1946)

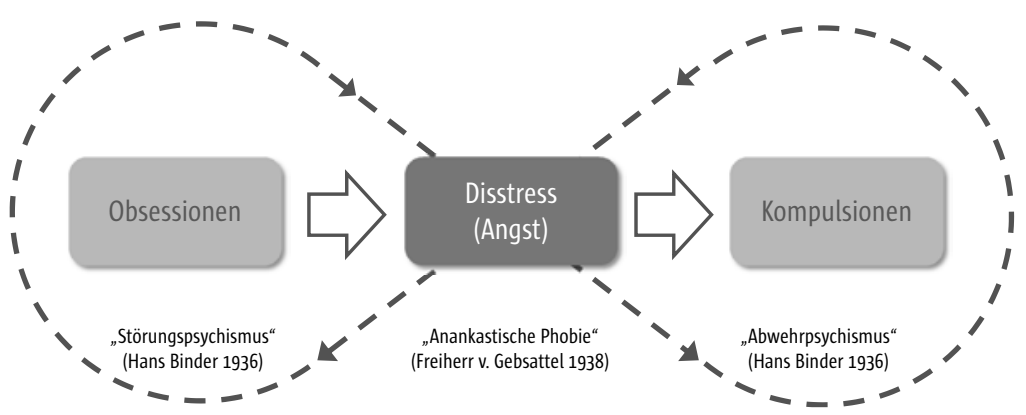

Abb. 35 Vereinfachtes Modell der strukturdynamischen Aspekte des Zwangssyndroms 
Im „Unrichtigkeitsbewusstsein“ sah Schneider (1925) schließlich das zentrale Kriterium, durch welches Zwangsphänomene von überwertigen oder wahnhaften Ideen abgegrenzt werden können.

\subsection{Die Zwangsstörung}

1980 erfuhr die Definition der Zwangsstörung im DSM-III (Diagnostic and Statistical Manual of Mental Disorders - Third Edition, APA 1980) erstmals eine kriterienbasierte Operationalisierung, welche bis in die Gegenwart in den gültigen Klassifikationssystemen für psychische Erkrankungen beibehalten wurde. Das wesentliche Kennzeichen der Störung ist das Vorliegen wiederkehrender Zwangsphänomene, die schwer genug sind, um eine bedeutsame leidvolle Beeinträchtigung oder eine merkliche psychosoziale Behinderung zu verursachen. Gemäß den Kriterien der ICD-10 (Dilling 2006) muss die Kernsymptomatik dabei für einem Zeitraum von mindestens zwei Wochen vorliegen, wobei an den meisten Tagen Zwangserscheinungen auftreten müssen, um die Diagnose stellen zu können (s. Tab. 11). Die Kriterien des DSM-IV-TR(APA 200o) ent-

\section{Tab. 11 Diagnostische Kriterien der Zwangsstörung gemäß ICD-10 (Dilling et al. 2006)}

\section{Zwangsstörung (F42)}

A Entweder Zwangsgedanken oder Zwangshandlungen (oder beides) an den meisten Tagen über einen Zeitraum von mindestens 2 Wochen.

B Die Zwangsgedanken (Ideen oder Vorstellungen) und Zwangshandlungen zeigen sämtliche folgende Merkmale:

1. Sie werden als eigene Gedanken/Handlungen von den Betroffenen angesehen und nicht als von anderen Personen oder Einflüssen eingegeben

2. Sie wiederholen sich dauernd und werden als unangenehm empfunden und mindestens ein Zwangsgedanke oder eine Zwangshandlung werden als übertrieben und unsinnig erkannt.

3. Die Betroffenen versuchen, Widerstand zu leisten (bei lange bestehenden Zwangsgedanken und Zwangshandlungen kann der Widerstand allerdings sehr gering sein). Gegen mindestens einen Zwangsgedanken oder eine Zwangshandlung wird gegenwärtig erfolglos Widerstand geleistet.

4. Das Ausführen eines Zwangsgedankens oder einer Zwangshandlung ist für sich genommen nicht angenehm (dies sollte von einer vorübergehenden Erleichterung von Anspannung oder Angst unterschieden werden).

C Die Betroffenen leiden unter den Zwangsgedanken und Zwangshandlungen oder werden in ihrer sozialen oder individuellen Leistungsfähigkeit behindert, meist durch den besonderen Zeitaufwand.

D Ausschlussvorbehalt: Die Störung ist nicht bedingt durch eine andere psychische Störung, wie Schizophrenie und verwandte Störungen (F2) oder affektive Störungen (F3).

Die Diagnose kann mit der folgenden vierten Stelle differenziert werden:

F42.0 vorwiegend Zwangsgedanken und Grübelzwang

F42.1 vorwiegend Zwangshandlungen (Zwangsrituale)

F42.2 Zwangsgedanken und -handlungen, gemischt

F42.8 sonstige Zwangsstörungen

F42.9 nicht näher bezeichnete Zwangsstörung 
halten diesbezüglich kein Zeitkriterium, wenngleich festgehalten wird, dass die Zwangsgedanken (obsessions) und Zwangshandlungen (compulsions) zeitaufwendig sein müssen, um als diagnoserelevant zu gelten (s. Tab. 11).

Einen Überblick über die Diagnosekriterien der Obsessive-Compulsive Disorder (OCD) gemäß DSM-IV-TR (APA 200o) gibt Tabelle 12.

Auf psychopathologischer Ebene wird in einer formalen Dimension übereinstimmend zwischen Zwangsgedanken und Zwangshandlungen differenziert (s. Abb. 36). Zwangsgedanken oder Obsessionen sind unwillkürlich und wiederholt auftretende, in den Gedankenstrom drängende und in diesem persistierende Ideen, die in Form von aufdringlichen Gedanken, bildhaften Vorstellungen oder dranghaften Impulsen Inhalte repräsentieren, die mit dem Selbst-

\section{Tab. 12 Auszug der diagnostischen Kriterien der Obsessive-Compulsive Disorder gemäß DSM-IV-TR (APA 2000) in Originalfassung}

Obsessive-Compulsive Disorder (300.3)

A Presence of obsessions, compulsions, or both:

Obsessions are defined by (1), (2), (3) and (4):

1. Recurrent and persistent thoughts, impulses, or images that are experienced, at some time during the disturbance, as intrusive and inappropriate, and that cause marked anxiety or distress.

2. The thoughts, impulses, or images are not simply excessive worries about real life problems.

3. The person attempts to ignore or suppress such thoughts, urges, or images, or to neutralize them with some other thought or action.

4. The person recognizes that the obsessional thoughts, impulses, or images are a product of his or her own mind (not imposed from without as in thought insertion).

Compulsions are defined by (1) and (2):

1. Repetitive behaviors (e.g., hand washing, ordering, checking) or mental acts (e.g., praying, counting, repeating words silently) that the individual feels driven to perform in response to an obsession or according to rules that must be applied rigidly.

2. The behaviors or mental acts are aimed at preventing or reducing anxiety or distress, or preventing some dreaded event or situation; however, these behaviors or mental acts are not connected in a realistic way with what they are designed to neutralize or prevent, or are cleanly excessive.

B At some point during the course of the disorder, the person has recognized that the obsessions or compulsions are excessive or unreasonable.

C The obsessions or compulsions cause marked distress, are time-consuming (e.g., take more than 1 hour per day), or significantly interfere with the person's normal routine, occupational (or academic) functioning, or usual social activities or relationships.

D If another Axis I disorder is present, the content of the obsessions or compulsions is not restricted to it (...).

E The disturbance is not due to the physiological effects of a substance (e.g., a drug of abuse, a medication) or general medical condition.

Specify if:

With poor insight: If, for most of the time during the current episode, the person does not recognize that the obsessions and compulsions are excessive or unreasonable. 


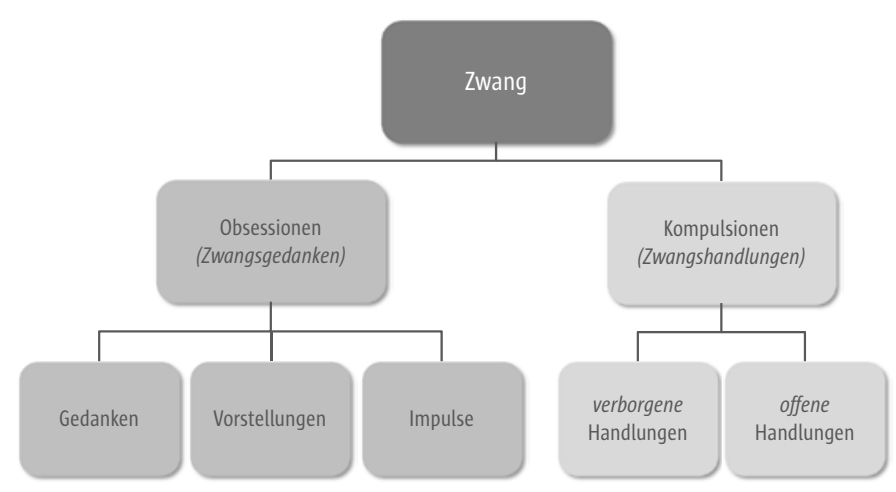

Abb. 36 Formale Dimension von Zwangsphänomenen

bild oder den moralischen Wertvorstellungen des Betroffenen zumeist unvereinbar sind. Aufgrund des formalen Kriteriums der Intrusivität sowie des inhaltlichen Kriteriums der Unannehmbarkeit, werden Obsessionen als störend, unangenehm, abstoßend, ekelerregend, quälend oder zumindest sinnlos erlebt und von Gefühlen eines Disstress, wie Unbehagen, Angst oder Ekel, begleitet (Zaudig 2011a; Kapfhammer 2008). Es können dabei drei Untergruppen von obsessiven Gedanken unterschieden werden (Zaudig 2011a):

1. zwanghaftes Zweifeln

2. zwanghafte Impulse

3. zwanghafte Vorstellungen und Bilder

Zwangshandlungen oder Kompulsionen sind häufig ritualisierte, zum Teil stereotype Handlungsabläufe, die immer gegen einen inneren Widerstand ausgeführt werden und sich vom Betroffenen nicht oder nur schwer unterbinden lassen, obwohl sie als unsinnig, übertrieben oder nicht zielführend erlebt werden (Zaudig 2011a; Kapfhammer 2008). Wird eine kompulsive Handlung unterlassen, führt dies in der Regel zu einem Anstieg von Anspannung oder Angst. Zwangshandlungen manifestieren sich in offener und somit beobachtbarer Form häufig als Waschen, Putzen, Sammeln, Kontrollieren, Ordnen oder Zählen. Verborgene Kompulsionen entsprechen mentalen Ritualen oder dem Zwangsdenken, wobei die formalen Kriterien von Zwangshandlungen erfüllen werden.

Während ICD-1o (Dilling et al. 2006) und DSM-IV-TR (APA 200o) Zwangsphänomene weitestgehend einheitlich definieren, bestehen hinsichtlich der Bewertung ihres dynamischen Verhältnisses deutliche Unterschiede. Die ICD-10 (Dilling et al. 2006) unterscheidet ausschließlich nach der extensionalen Zugehörigkeit der Zwangssymptome, wobei sich Obsessionen und Kompulsionen in einem statischen und äquivalenten Verhältnis zueinander befinden (Kapfhammer 2008). Zwangsgedanken werden dabei der „,mentalen Sphäre“ und Zwangshandlungen der „behavioralen Sphäre“ zugerechnet. Das DSM-IV (TR) betont hingegen das dynamische Verhältnis zwischen beiden Phänomenen und stellt klar, dass „obsessions“ zu einem emotionalen Disstress führen, auf dessen 
Kontrolle „compulsions“ in Form kognitiver oder behavioraler Akte abzielen. Zwangshandlungen umfassen somit sowohl beobachtbare als auch mentale Aktivitäten, die darauf ausgerichtet sind, die durch Zwangsgedanken ausgelöste Angst zu reduzieren. Gemäß der ICD-10 müssten somit mentale Rituale und Zwangsdenken grundsätzlich als Zwangsgedanken bewertet werden, während diese nach DSM-IV-TR den Kompulsionen, nämlich den verborgenen Zwangshandlungen, zugeordnet werden können (s. Abb. 37). Nach Kapfhammer stellt diese Konzeptualisierung nicht einfach eine arbiträre psychopathologische Akzentverschiebung dar, sondern das dynamische Verhältnis gemäß DSM-IV-TR erscheint besonders im Hinblick auf eine Therapieplanung auch schlüssiger angelegt (Kapfhammer 2008). Ferner wird es aufgrund der angeführten Grundannahmen auch verständlich, weshalb in der ICD-10 eine Subtypisierung in: „Vorwiegend Zwangsgedanken oder Grübelzwang (F42.o)“, „Vorwiegend Zwangshandlungen (F42.1)“ sowie „Zwangsgedanken und -handlungen, gemischt (F42.2)“ möglich ist, während diese im DSM-IV (TR) nicht angeführt wird.

Die affektive Konnotation von Zwangsphänomenen als zumindest unangenehm, quälend und angstbesetzt, wird schließlich einheitlich als definitorisches Kriterium hervorgehoben, wodurch auch eine kategoriale Abgrenzung zu anderen impulsgeleiteten oder dranghaften Verhaltensweisen ermöglicht wird. Das DSM-IV-TR sah in der Zwangsstörung dabei einen Prägnanztyp der Angststörung, während die ICD-10 das obsessiv-kompulsive Syndrom als eigenständige Entität anderen Störungen aus dem neurotisch-belastungsreaktiven Formenkreis gegenüberstellt. Im März 2013 wurde von der Amerikanisch-psychiatrischen Vereinigung (APA) die fünfte Version des Diagnostischen und Statistischen Manuals Psychischer Störungen (DSM-5) veröffentlicht, welche zahlreiche Veränderungen hinsichtlich der Klassifikation seeli-

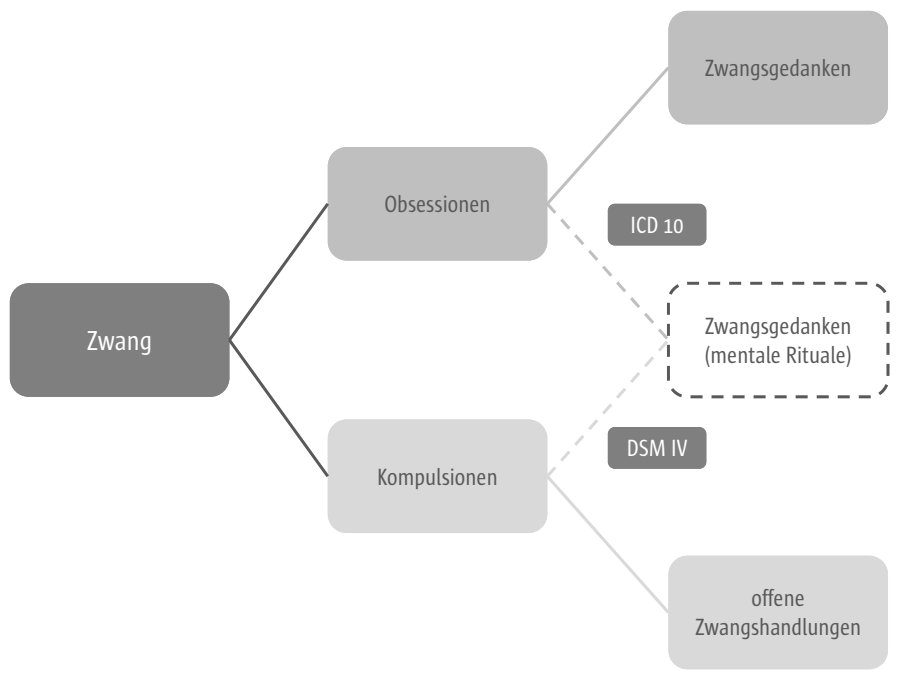


scher Erkrankungen beinhaltet. Die „Obsessive-Compulsive Disorder“ ist nunmehr keine Angststörung im engeren Sinne mehr, sondern befindet sich mit anderen, durch impulsiv-kompulsives Verhalten gekennzeichneten Störungsbildern, wie „Body Dysmorphic Disorder“, „Trichotillomania“, „Excoriation (Skin-Picking) Disorder“ und „Hoarding Disorder“, in einer eigenen Störungsgruppe, welche als „Obsessive-Compulsive and Related Disorders“ bezeichnet wurde. Die aktuellen, revidierten Diagnosekriterien für Zwangsstörungen gemäß DSM-5 (APA 2013) sind folgender Tabelle zu entnehmen (s. Tab. 13). Inwieweit diese Neufassung einem Erkenntnisgewinn dienlich sein könnte oder ob die Revision der Diagnosekriterien eine behindernde Ausweitung des Zwangsbegriffes darstellt, wird in den kommenden Jahren zweifellos Gegenstand eingehender Diskussionen werden.

\section{Tab. 13 Auszug der diagnostischen Kriterien der Obsessive-Compulsive Disorder gemäß DSM-5 (APA 2013) in Originalfassung}

\section{Obsessive-Compulsive Disorder (300.3)}

A Presence of obsessions, compulsions, or both:

Obsessions are defined by (1) and (2):

1. Recurrent and persistent thoughts, urges, or images that are experienced, at some time during the disturbance, as intrusive and unwanted, and that in most individuals cause marked anxiety and distress.

2. The individual attempts to ignore or suppress such thoughts, urges, or images, or to neutralize them with some other thought or action (i.e., by performing a compulsion).

Compulsions are defined by (1) and (2):

1. Repetitive behaviors (e.g., hand washing, ordering, checking) or mental acts (e.g., praying, counting, repeating words silently) that the individual feels driven to perform in response to an obsession or according to rules that must be applied rigidly.

2. The behaviors or mental acts are aimed at preventing or reducing anxiety or distress, or preventing some dreaded event or situation; however, these behaviors or mental acts are not connected in a realistic way with what they are designed to neutralize or prevent, or are cleanly excessive.

B The obsessions or compulsions are time-consuming (e.g., take more than 1 hour per day) or cause clinically significant distress or impairment in social, occupational, or other important areas of functioning.

C The obsessive-compulsive symptoms are not attributable to the physiological effects of a substance (e.g., a drug of abuse, a medication) or another medical condition.

D The disturbance is not better explained by the symptoms of another medical condition (...).

\section{Specify if:}

With good or fair insight: The individual recognizes that obsessive-compulsive disorder beliefs are definitely or probably not true or that they may or may not be true.

With poor insight: The individual thinks obsessive-compulsive disorder beliefs are probably true.

With absent insight/delusional beliefs: The individual is completely convinced that obsessive-compulsive disorder beliefs are true.

\section{Specify if:}

Tic-related: The individual has a current or past history of a tic disorder. 


\subsection{Phänomenologische Aspekte von Zwangsphänomenen}

Psychischer Zwang lässt sich in einer formalen Dimension als intrusives mentales Erlebnis in Form von Obsessionen und Kompulsionen auffassen (Kapfhammer 2008). Zwangsgedanken sind dabei durch wiederkehrende und persistierende Ideen charakterisiert, welche Unbehagen oder Angst verursachen, während Zwangshandlungen stereotype Rituale repräsentieren, die ausgeführt werden, um diese aversiven Gefühle zu reduzieren (Stompe 2011). Zwangsphänomene scheinen somit durchaus homogene Erscheinungen zu sein, so denn auch die internationalen operationalisierten Diagnosekriterien für Zwangsstörungen diese Einheitlichkeit vordergründig suggerieren. Tatsächlich verweist die obsessiv-kompulsive Störung jedoch auf durchaus heterogene klinische Syndrome. Nach Akhtar und Kollegen (1975) zählen dabei die Kategorien Schmutz und Kontamination, Aggression, Sexualität und Religion zu den häufigsten inhaltlichen Bereichen bei Zwangserscheinungen (Akhtar et al. 1975). In der DSM-IV Feldstudie konnten für den US-amerikanischen Bereich folgende Häufigkeiten von Obsessionen und Kompulsionen ermittelt werden (s. Tab. 14 u. 15).

\section{Tab. 14 Rangliste häufiger Inhalte von Obsessionen bei 431 Zwangskranken - nordamerikanische DSM-IV Feldstudie (modifiziert nach Foa et al. 1995)}

\begin{tabular}{l|c}
\hline obsessive Gedanken & Häufigkeit (\%) \\
\hline Kontamination & 37,8 \\
\hline Unglück und Schaden & 23,6 \\
\hline Ordnung und Symmetrie & 10,0 \\
\hline körperliche Beschwerden & 7,2 \\
\hline Religion & 5,9 \\
\hline Sexualität & 5,5 \\
\hline Horten und Sammeln & 4,8 \\
\hline Aggression und Gewalt & 4,3 \\
\hline
\end{tabular}

Tab. 15 Rangliste häufiger Kompulsionen bei 431 Zwangskranken - nordamerikanische DSM-IV Feldstudie (modifiziert nach Foa et al. 1995)

\begin{tabular}{l|c}
\hline kompulsive Handlungen & Häufigkeit (\%) \\
\hline Kontrollieren & 28,8 \\
\hline Reinigen und Waschen & 26,5 \\
\hline Wiederholen & 11,1 \\
\hline mentale Rituale & 10,9 \\
\hline Ordnen & 5,9 \\
\hline Horten und Sammeln & 3,5 \\
\hline Zählen & 2,1 \\
\hline
\end{tabular}


Durch zahlreiche moderne faktorenanalytische Studien konnten charakteristische Kombination von Zwangsgedanken und Zwangshandlungen nachgewiesen werden, welche als Symptomdimensionen bezeichnet werden (Kapfhammer 2008; Zaudig 2011b). Bloch und Kollegen (2008) konnten in einer Metaanalyse von 12 Faktoranalysen mit insgesamt mehr als 2000 Zwangspatienten dabei aufzeigen, dass beim obsessiv-kompulsiven Syndrom zumindest vier Zwangsdimensionen reliabel unterschieden werden können (Bloch et al. 2008) (s. Tab. 16).

Fullana und Kollegen (2010) untersuchten die Häufigkeit von Zwangssymptomen in sechs europäischen Nationen (Belgien, Deutschland, Frankreich, Holland, Italien und Spanien), wobei sie die Dimension „Verbotene Gedanken“ nach Bloch (2008) in vier Subgruppen unterteilten. Sie konnten dabei zeigen, dass hinsichtlich der Prävalenz in den untersuchten europäischen Staaten keine relevanten Unterschiede bestehen, wobei Frauen signifikant häufiger Zwangssymptome mit aggressivem und körperbezogenem Inhalt sowie Verunreinigungsängste und Waschzwänge aufweisen (s. Tab. 17).

Neben der charakteristischen Kombination von Zwangsgedanken und Zwangshandlungen, zeigen Symptomdimensionen auch neurobiologische Unter-

Tab. 16 Grunddimensionen obsessiv-kompulsiver Symptome (modifiziert nach Bloch et al. 2008)

\begin{tabular}{|l|l|}
\hline Dimension & Beschreibung \\
\hline Kontamination/Reinigung & $\begin{array}{l}\text { Verschmutzungsgedanken und Kontaminationsängste mit Wasch-, } \\
\text { Putz- und Reinigungszwängen }\end{array}$ \\
\hline Symmetrie/Ordnung & $\begin{array}{l}\text { Zwangsgedanken hinsichtlich Symmetrie, Ordnung und Genauigkeit } \\
\text { mit Ordnungs- und Wiederholungszwängen }\end{array}$ \\
\hline Horten & Obsessionen und Kompulsionen hinsichtlich Sammeln und Horten \\
\hline „verbotene Gedanken“ & $\begin{array}{l}\text { aggressive, sexuelle, religiöse sowie körperbezogene Zwangsgedanken } \\
\text { mit Kontrollzwängen oder mentalen Ritualen }\end{array}$ \\
\hline
\end{tabular}

Tab. 17 Rangfolge von sieben Zwangsdimensionen entsprechend der Lebenszeitprävalenz (\%) in der europäischen Allgemeinbevölkerung ${ }^{1}$ (modifiziert nach Fullana et al. 2010)

\begin{tabular}{lcccc}
\hline Dimension & gesamt (\%) & Männer (\%) & Frauen (\%) & $p^{2}$ \\
\hline Schädigung und Aggression & 7,8 & 5,6 & 9,9 & $<0,01$ \\
\hline Körper und Erkrankung & 4,6 & 2,5 & 6,5 & $<0,001$ \\
\hline Ordnung und Symmetrie & 3,1 & 2,0 & 4,0 & n.s. \\
\hline Sammeln und Horten & 2,6 & 2,2 & 2,9 & n.s. \\
\hline $\begin{array}{l}\text { Verunreinigung } \\
\text { Kontamination }\end{array}$ & 1,8 & 0,7 & 2,9 & $<001$ \\
\hline moralische Normen & 1,4 & 1,4 & 1,4 & n.s. \\
\hline Sexualität und Religion & 0,7 & 0,9 & 0,6 & n.s. \\
\hline
\end{tabular}

${ }^{1}$ Stichprobengröße N = 2.804 (6 europäische Länder: Belgien, Deutschland, Frankreich, Holland, Italien, Spanien)

${ }^{2}$ Signifikanzwert p: „n.s.“ = nicht signifikant 
schiede (Matiax-Cols et al. 2005). So können bei Waschzwängen und Kontaminationsängsten höhere Aktivitäten im Bereich des orbitofrontalen Kortex und der Amygdala nachgewiesen werden, während bei Kontrollzwängen der Bereich des anterioren zingulären Kortex stärker aktiv ist (Zaudig 2011b).

Eine Subtypisierung anhand der vorherrschenden Symptomatik erscheint auch deshalb sinnvoll, da von ethnobiologischer und ethologischer Seite angenommen wird, dass Zwangsphänomene phylogenetisch ältere Verhaltensprogramme repräsentieren, die auf den Schutz vor speziellen Bedrohungen ausgerichtet sind. Die beobachtbaren Symptome bei Zwangsstörungen werden dabei als Ausdruck der Aktualisierung oder Disinhibition archaischer Hirnstrukturen angesehen, welche durch eine geschwächte Kontrolle von höheren (neokortikalen) Strukturen demaskiert und verhaltensbestimmend werden (Kohl 1998). Nach Süllwold (2006) verhält sich etwa ein Zwangskranker mit Waschzwängen durch das Hervortreten solcher Instinktschemata analog zu einem Urmenschen, der ein bestimmtes Areal von Spuren freizuhalten zu versucht. Es scheinen somit deutliche Ähnlichkeiten zwischen krankhaftem Zwangsverhalten und archaischem Territorialverhalten zu bestehen, wobei vor allem Kontaminationsängste und Symmetriebedürfnisse auf Urängste vor Krankheit und Reviergefährdung verweisen (Kohl 1998; Süllwold 2006). Die paläopsychiatrische Ansicht, dass obsessiv-kompulsive Symptome grundsätzlich überindividuelle Verhaltensaspekte der Phylogenese darstellen, welche durch individuell-lerngeschichtliche Komponenten eine "pathoplastische“ Tönung erhalten, wird schließlich durch Studienergebnisse gestützt, welche belegen, dass Zwangssymptome bzw. -dimensionen eine kulturübergreifende Homogenität aufweisen (Kohl 1998; Zaudig 2011a).

In einer spezifisch inhaltlichen Dimension ist für Zwangsphänomene charakteristisch, dass in ihnen Aspekte von kontaminierendem Schmutz, unkalkulierbarer Gefahr, erschreckender Sexualität, bedrohlicher Aggression, Verstößen gegen moralische Standards, gesellschaftliche Normen und religiöse Gebote aufscheinen. Sie verweisen nach Kapfhammer (2008) somit auf eine inhärente Verbindung von Zwang zu einer als bedroht erlebten persönlichen, moralischen und sozialen Werteordnung und legen einen Zusammenhang zu soziokulturellen Einflüssen nahe (Kapfhammer 2008). Kultur beeinflusst die Gestaltung psychopathologischer Phänomene dabei durch Symbolsysteme, die im Rahmen der Ontogenese eines Individuums durch sozialisierende Prozesse internalisiert werden. In den Inhalten und Themen der Zwangsphänomene kommen somit Leitbilder und Ängste einer Kultur in Form einer epochalen Bedingtheit (Pfeiffer 1994) zum Ausdruck. Dementsprechend kann ein soziokultureller Wandel auch mit einer Veränderung der Zwangsthematik verbunden sein.

\subsection{Epidemiologie}

Nach aktueller Schätzung des European Brain Council (EBC) und des European College of Neuropsychopharmacology (ECNP) waren 2010 in der Gesamtbevöl- 
kerung der 27 EU-Mitgliedstaaten, der Schweiz, Norwegens und Islands insgesamt rund 2, 9 Millionen Personen von einer Zwangsstörung betroffen. Dies entspricht einer mittleren 12-Monatsprävalenz von $0,7 \%$, wobei von der Forschungsgruppe nach Analyse von 10 epidemiologischen Studien aus Europa ein Vertrauensbereich von $0,5 \%$ bis $1,1 \%$ berechnet werden konnte (Wittchen et al. 2011). Im Vergleich zu den Angststörungen, deren mittlere Jahresprävalenz in Europa zwischen 1\% und 7\% geschätzt wird, sind Zwangsstörungen in der Allgemeinbevölkerung somit eher selten vorzufinden. Dennoch zählen sie zu den häufigsten neuropsychiatrischen Ursachen einer beeinträchtigten Lebensqualität, da sowohl die Verheimlichungstendenz der Betroffenen als auch die mangelhafte psychiatrische Versorgung zu einer erheblichen Behandlungsverzögerung und somit zu einer Zunahme der psychosozialen Behinderungsdauer führen.

Bis in die achtziger Jahre des letzten Jahrhunderts wurde in der Fachwelt die Ansicht vertreten, dass es sich bei der Zwangsstörung um ein äußerst seltenes seelisches Krankheitsbild handeln würde (Zaudig 2011a; Kapfhammer 2008; Vorderholzer et al. 2011). Mit der Etablierung operationalisierter Diagnosekriterien, der Entwicklung standardisierter Erhebungsinstrumente sowie der Durchführung epidemiologischer Feldstudien sollte sich diese Ansicht jedoch grundlegend ändern. Zu den international am häufigsten zitierten Studien dieser Zeit zählt die Epidemiologic Catchment Area Study (ECA) des National Institutes of Mental Health (NIMH). In dieser Studie wurden zwischen 1980 und 1984 knapp 19.ooo erwachsene Personen in 5 Regionen der USA (New Haven/Connecticut, Baltimore/Maryland, Saint Louise/Missouri, Piedmont/ North Carolina, Los Angeles/Kalifornien) untersucht, wobei erstmals ein vollstrukturiertes Erhebungsinstrument für Laieninterviews, das Diagnostic Interview Schedule (DIS), zur Anwendung kam, das von Lee Roberts und Kollegen an der Washington University entwickelt wurde (Eaton et al. 1981; Regier et al. 1984). Die Diagnose einer Zwangsstörung wurde dabei gemäß den Kriterien des DSM-III (APA 1980) gestellt, wobei eine 6-Monatsprävalenz von 1,5\%, eine 1 -Jahresprävalenz von 1,6\% sowie eine Lebenszeitprävalenz von $2,5 \%$ berechnet werden konnten (s. Abb. 38). Mit diesen Ergebnissen fand sich die Zwangsstörung in der Rangfolge der häufigsten psychischen Störungen in der Bevölkerung nach den Phobien, dem Substanzmissbrauch und der Depression an vierter Stelle wieder (Karno et al. 1988).

Aufgrund der Breitenwirkung der ECA-Ergebnisse wurden in den Folgejahren zahlreiche Feldstudien mittels DIS in unterschiedlichen Nationen durchgeführt. In der Cross National Collaborative Study der 199oer-Jahre, in der Weissmann und Kollegen (1994) epidemiologische Studien aus 6 Nationen (USA/ Amerika, Puerto Rico/Amerika, Kanada/Amerika, Südkorea/Asien, Taiwan/ Asien, Neuseeland/Ozeanien) zusammenfassten, konnte hinsichtlich der Häufigkeitsraten eine bemerkenswerte Übereinstimmung festgestellt werden. Allein die Prävalenzraten aus Taiwan waren deutlichen niedriger (s. Abb. 39). 


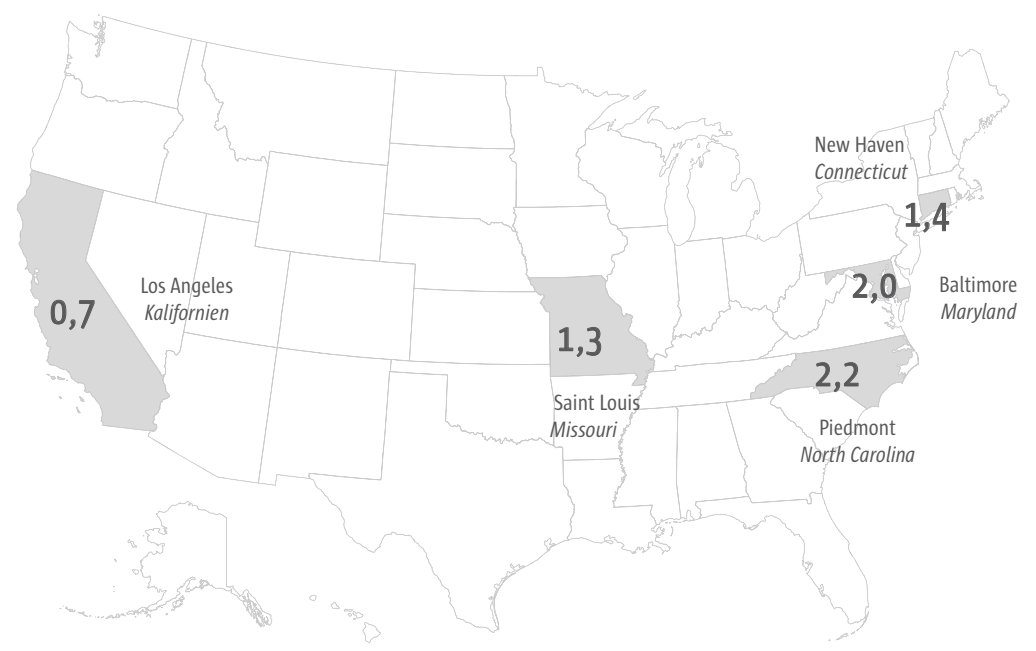

Abb. 38 6-Monats-Prävalenz (\%) der Obsessive-Compulsive Disorder (DIS/DSM-III) in fünf USamerikanischen Regionen - ECA Studie des NIMH (modifiziert nach Karno et al. 1988)

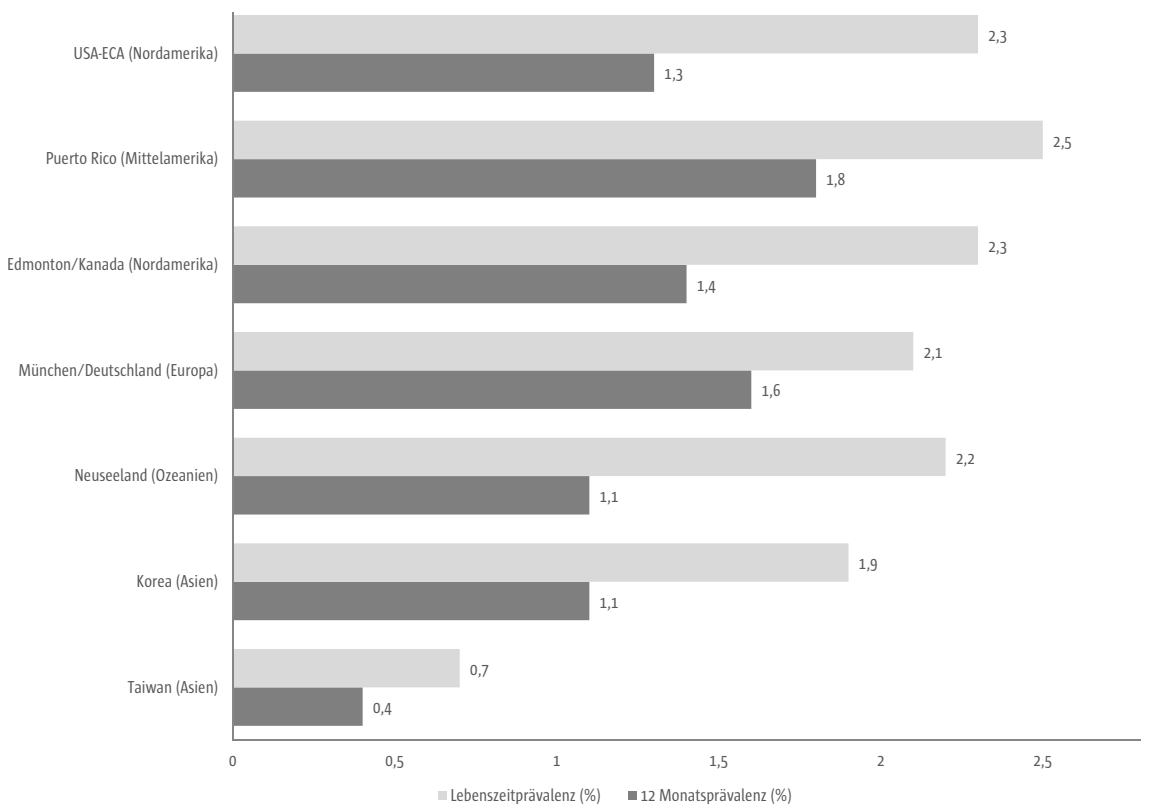

Abb. 39 Periodenprävalenz (\%) der Obsessive-Compulsive Disorder (DIS/DSM-III) im internationalen Vergleich - Cross National Collaborative Group (modifiziert nach Weissman et al. 1994)

Die zwischen 2001 und 2003 durchgeführte Replikationsstudie des National Comorbidiy Surveys (NCS-R), in welcher ein Subsample von insgesamt 2073 erwachsenen Personen der amerikanischen Bevölkerung mittels des World Health Organization Composite International Diagnostic Interviews (CIDIVersion 3.0) hinsichtlich dem Vorliegen einer obsessiv-kompulsiven Störung 
gemäß DSM-IV untersucht wurde, konnte mit einer Lebenszeithäufigkeit von 2,3\% und einer 12-Monatsprävalenz von 1,2\% die ECA-Prävalenzraten für den amerikanischen Raum bestätigen (Ruscio et al. 2010). Für Europa konnten Wittchen et al. (2005) in einer Zusammenfassung von 9 epidemiologischen Studien und einer Samplegröße von nahezu 40.0oo Personen jedoch lediglich eine mittlere Jahresprävalenz von o,7\% (Quartilsabstand o,5\%-1,1\%) aufzeigen. Grabe und Kollegen konnten in einer Untersuchung an 4.075 Erwachsenen aus der deutschen Bevölkerung ebenfalls ein geringeres Vorkommen der Zwangsstörung (Lebenszeitprävalenz 0,5\%, Jahresprävalenz 0,4\%) als in den Vereinigten Staaten nachweisen (Grabe et al. 2000; Grabe et al. 2001).

Prävalenzraten der Zwangsstörung in sieben Regionen der Welt zeigen, dass hinsichtlich der Häufigkeit von Zwangsstörung sowohl deutliche interregionale als auch innerregionale Unterschiede bestehen, welche wohl zu einem Großteil auf die unterschiedlichen Diagnosekriterien und Erhebungsinstrumente sowie die Stichprobenzusammensetzung zurückgeführt werden können (s. Tab. 18).

\section{Tab. 18 Periodenprävalenz (\%) der Zwangsstörung in der Allgemeinbevölkerung - internationaler Vergleich anhand ausgewählter Studien}

\begin{tabular}{|c|c|c|c|c|}
\hline Nation & Referenz & Diagnostik & \multicolumn{2}{|c|}{ Prävalenzraten (\%) } \\
\hline $\begin{array}{l}\text { (Region/Studie, Erhebungs- } \\
\text { zeitraum) }^{1}\end{array}$ & & $\begin{array}{l}\text { Instrument/ } \\
\text { Kriterien }^{2}\end{array}$ & 12-Monate ${ }^{3}$ & Lebenszeit ${ }^{3}$ \\
\hline \multicolumn{5}{|l|}{ AMERIKA } \\
\hline \multicolumn{5}{|l|}{ Nordamerika } \\
\hline Kanada (Edmonton, 1983-1986) & Bland et al. 1988a, 1988b & DIS/DSM-III & 1,8 & 3,0 \\
\hline USA (ECA, 1980-1984) & Karno et al. 1988 & DIS/DSM-III & 1,6 & 2,5 \\
\hline USA (NCS-R, 2001-2003) & Ruscio et al. 2010 & CIDI/DSM-IV & 1,2 & 2,3 \\
\hline \multicolumn{5}{|l|}{ Lateinamerika und Karibik } \\
\hline Brasilien (São Paulo) & Andrade et al. 2002 & CIDI/ICD-10 & 0,3 & 0,3 \\
\hline Brasilien (SPMHS, 2005-2007) & $\begin{array}{c}\text { Andrade et al. 2012; Viana u. } \\
\text { Andrade } 2012\end{array}$ & CIDI/DSM-IV & 3,9 & 6,7 \\
\hline Chile (CPPS, 1992-1999) & Vicente et al. 2006 & CIDI/DSM-III-R & 1,2 & 1,2 \\
\hline Mexiko (Mexico City, 1995) & $\begin{array}{l}\text { Caraveo-Anduaga u. Bermudez } \\
2004\end{array}$ & CIDI/ICD-10 & 1,0 & 1,4 \\
\hline Puerto Rico (Puerto Rico, 1984) & Canino et al. 1987 & DIS/DSM-III & - & 3,2 \\
\hline \multicolumn{5}{|l|}{ EUROPA } \\
\hline Deutschland (TACOS, 1996-1997) & $\begin{array}{l}\text { Grabe et al. 2000; Grabe et al. } \\
\qquad 2001\end{array}$ & CIDI/DSM-IV & 0,4 & 0,5 \\
\hline Deutschland (GHS-MHS, 1996-1997) & $\begin{array}{l}\text { Jacobi et al. 2004; Adam et al. } \\
2011\end{array}$ & CIDI/DSM-IV & 0,7 & - \\
\hline Irland (NISHS, 2004-2008) & Bunting et al. 2012 & CIDI/DSM-IV & - & 0,5 \\
\hline Island (Island, 1931) & Stefansson et al. 1991 & DIS/DSM-III & - & 2,0 \\
\hline Italien (Florenz, 1984) & Faravelli et al. 1989 & SADS/DSM-III & - & 0,7 \\
\hline Niederlande (NEMESIS, 1996) & Bijl et al. 1998 & CIDI/DSM-III-R & 0,5 & 0,9 \\
\hline
\end{tabular}




\section{Zwangsstörungen und Kultur}

\begin{tabular}{|c|c|c|c|c|}
\hline \multirow{2}{*}{$\begin{array}{l}\text { Nation } \\
\text { Norwegen (Oslo, 1994-1997) }\end{array}$} & \multirow{2}{*}{$\begin{array}{c}\text { Referenz } \\
\text { Kringlen et al. } 2001\end{array}$} & \multirow{2}{*}{$\begin{array}{l}\text { Diagnostik } \\
\text { CIDI/DSM-III-R }\end{array}$} & \multicolumn{2}{|c|}{ Prävalenzraten (\%) } \\
\hline & & & 0,7 & 1,6 \\
\hline Rumänien (RMHS, 2005-2007) & Florescu et al. 2009 & CIDI/DSM-IV & - & 0,6 \\
\hline Schweiz (ZCS, 1979-1999) & $\begin{array}{l}\text { Dagonda et al. 1993; Angst } \\
\text { et al. } 2004\end{array}$ & $\begin{array}{l}\text { SPIKE/ } \\
\text { DSM-III-R }\end{array}$ & 0,7 & 3,5 \\
\hline Ungarn (Ungarn, 1995-1996) & Nemeth et al. 1997 & DIS/DSM-III-R & - & 2,7 \\
\hline \multicolumn{5}{|l|}{ ASIEN } \\
\hline \multicolumn{5}{|l|}{ West- und Südwestasien } \\
\hline Irak (IMHS, 2006-2007) & Alhasnawi et al. 2009 & CIDI/DSM-IV & 3,6 & 4,6 \\
\hline Iran (Iran, 2001) & Mohammadi et al. 2004 & SADS/DSM-IV & - & 1,8 \\
\hline Libanon (LEBANON, 2002-2003) & Karam et al. 2006 & CIDI/DSM-IV & 0,1 & - \\
\hline Türkei (Konya, 2000) & Cilli et al. 2004 & CIDI/DSM-IV & 3,0 & - \\
\hline VAE (Al Ain City, 1996-1997) & Abou-Saleh et al. 1997 & CIDI/ICD-10 & - & 0,1 \\
\hline \multicolumn{5}{|l|}{ Ost- und Südostasien } \\
\hline China (SCMHS, 1984-1986) & Chen et al. 1993 & DIS/DSM-III & - & 1,0 \\
\hline China (Taiwan) (TPEP, 1982-1986) & Hwu et al. 1989 & DIS/DSM-III & $0,1^{*}$ & $0,5^{*}$ \\
\hline Korea (Seoul u. Myeon 1984) & Lee et al. 1987 & DIS/DSM-III & - & 2,1 \\
\hline Korea (KECA, 2001) & Cho et al. 2007 & CIDI/DSM-IV & 0,6 & 0,8 \\
\hline Singapur (SMHS, 2009-2010) & Subramaniam et al. 2012 & CIDI/DSM-IV & 1,1 & 3,0 \\
\hline \multicolumn{5}{|l|}{ AFRIKA } \\
\hline Nigeria (NSMHW, 2001-2003) & Gureje et al. 2006 & CIDI/DSM-IV & 0,1 & 0,1 \\
\hline \multicolumn{5}{|l|}{ OZEANIEN } \\
\hline Australien (NSMHWB, 1997) & $\begin{array}{l}\text { Andrews et al. 2001; Crino } \\
\text { et al. } 2005\end{array}$ & CIDI/DSM-IV & 0,7 & - \\
\hline Australien (NSMHWB, 2007) & McEvoy et al. 2011 & CIDI/DSM-IV & 2,7 & 3,8 \\
\hline Neuseeland (CPES, 1986) & $\begin{array}{l}\text { Oakley Browne et al. 1989; } \\
\text { Wells et al. } 1989\end{array}$ & DIS/DSM-III & 1,1 & 2,2 \\
\hline Neuseeland (NZMHS, 2003-2004) & $\begin{array}{l}\text { Oakley Browne et al. 2006; } \\
\text { Wells et al. } 2006\end{array}$ & CIDI/DSM-IV & 0,6 & 1,2 \\
\hline
\end{tabular}

* Angegebene Prävalenzraten entsprechen dem Median der publizierten Daten (Hwu et al. 1989)

${ }^{1}$ AACPS = Al Ain Community Psychiatric Survey; CPES = Christchurch Psychiatric Epidemiology Study; CPPS = Chile Psychiatric Prevalence Study; ECA = Epidemiologic Catchment Area; GHS-MHS = German National Health Interview and Examination Survey; IMHS = Iraq Mental Health Survey; KECA = Korean Epidemiologic Catchment Area Study; LEBANON = Lebanese Evaluation of the Burden of Ailments and Needs of the Nation; NCS-R = National Comorbidity Survey-Revised; NEMESIS = Netherlands Mental Health Survey and Incidence Study; NISHS = Northern Ireland Study of Health and Stress; NSMHW = Nigerian Survey of Mental Health and Well-Being; NSMHWB = Australian National Survey of Mental Health and Wellbeing; NZMHS = New Zealand Mental Health Survey (Te Rau Hinengaro); RMHS = Romanian Mental Health Study; SCMHS = Shatin Community Mental Health Survey; SMHS = Singapore Mental Health Survey; SPMHS = São Paulo Megacity Mental Health Survey; TACOS = Transitions in Alcohol Consumption and Smoking (Schleswig Holstein); TPEP = Taiwan Psychiatric Epidemiological Project; VAE = Vereinigte Arabische Emirate; ZCS = Zurich Cohort Study.

${ }^{2} \mathrm{CIDI}=$ Composite International Diagnostic Interview; CIS-R = Clinical Interview Schedule-Revised; DIS = Diagnostic Interview Schedule; DSM-III = Diagnostic and Statistical Manual of mental disorders, third edition; DSM-III-R = Diagnostic and Statistical Manual of mental disorders, third edition, revised; DSM-IV = Diagnostic and Statistical Manual of mental disorders, fourth edition; ICD-10 = International Classification of Diseases, , tenth revision; MINI = Mini International Neuro-psychiatric Interview; SADS = Schedule for Affective Disorders and Schizophrenia; SCAN = Schedule for Clinical Assessment in Neuropsychiatry; SPIKE = Structured Psychopathological Interview and Rating of the Social Consequences for Epidemiology.

3 Zeichenerklärung: ,„“" = nicht verfügbar 


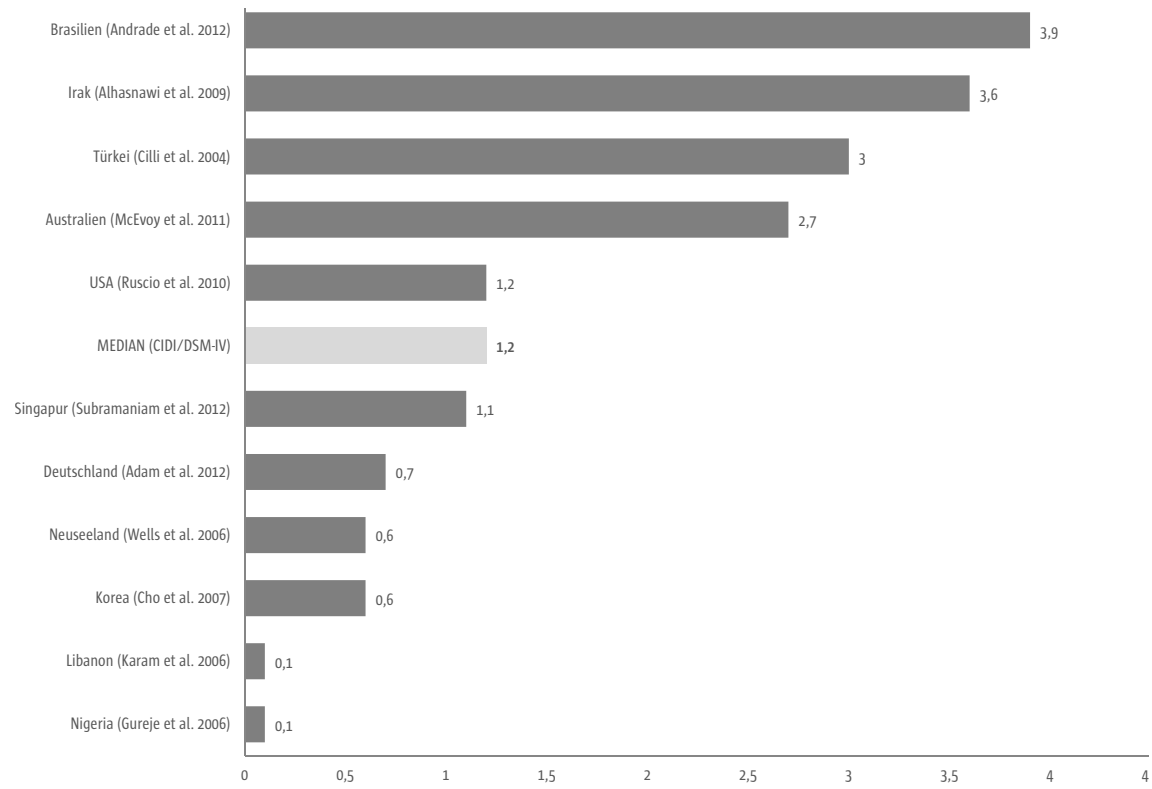

Abb. 40 Jahresprävalenz (\%) der Zwangsstörung (CIDI/DSM-IV) in der Allgemeinbevölkerung internationaler Vergleich anhand ausgewählter Studien

In einem Vergleich rezenter CIDI/DSM-IV Studien aus 11 Nationen zeigt sich dabei eine globale Varianz der Jahresprävalenz für Zwangsstörungen zwischen $0,1 \%$ und $3,9 \%$. Nahe am errechneten Median $(1,2 \%)$ finden sich die USA $(1,2 \%)$ und Singapur $(1,1 \%)$. Australien $(2,7 \%)$, Türkei $(3,0 \%)$, Irak $(3,6 \%)$ und Brasilien $(3,9 \%)$ zeigen deutlich höhere Prävalenzen, während Deutschland $(0,7 \%)$, Neuseeland $(0,6 \%)$ und Süd-Korea $(0,6 \%)$ sowie schließlich der Libanon $(0,1 \%)$ und Nigeria (o,1\%) geringere Häufigkeitsraten aufweisen (s. Abb. 40).

In vorwiegend individualistisch orientierten Regionen beträgt die Jahresprävalenz, ungeachtet der unterschiedlichen Diagnosekriterien und Erhebungsinstrumente, zwischen $0,6 \%$ und 1,8\%, wobei Zwangsstörungen in nordamerikanischen Ländern (1,2\%-1,8\%) und Ozeanien (o,6\%-2,7\%) im Durchschnitt häufiger diagnostiziert werden als in Europa $(0,5 \%-0,7 \%)$. In vorwiegend kollektivistischen und traditionellen Regionen beträgt der entsprechende Häufigkeitsbereich $0,1 \%$ bis $3,9 \%$, wobei lateinamerikanische $(1,2 \%-3,9 \%)$ und westasiatische $(0,1 \%-3,6 \%)$ Nationen höhere Prävalenzraten aufweisen als ostasiatische $(0,1 \%-1,1 \%)$ oder afrikanische $(0,1 \%)$ Länder (s. Abb. 41).

Somers und Kollegen (2006) konnten in einer Übersichtsarbeit folgende Erwartungswerte für die Periodenprävalenzen von Zwangsstörungen berechnen:

1. globale Prävalenzraten: Jahresprävalenz o,5\% (95\% Konfidenzintervall: o,3\%0,9\%), Lebenszeitprävalenz 1,3\% (95\% Konfidenzintervall: 0,9\% bis 1,8\%)

2. asiatische Länder: Jahresprävalenz $0,2 \%$ ( $95 \%$ Konfidenzintervall: $0,1 \%$ 0,3\%), Lebenszeitprävalenz 0,9\% (95\% Konfidenzintervall: $0,5 \%$ bis $1,5 \%$ ) 


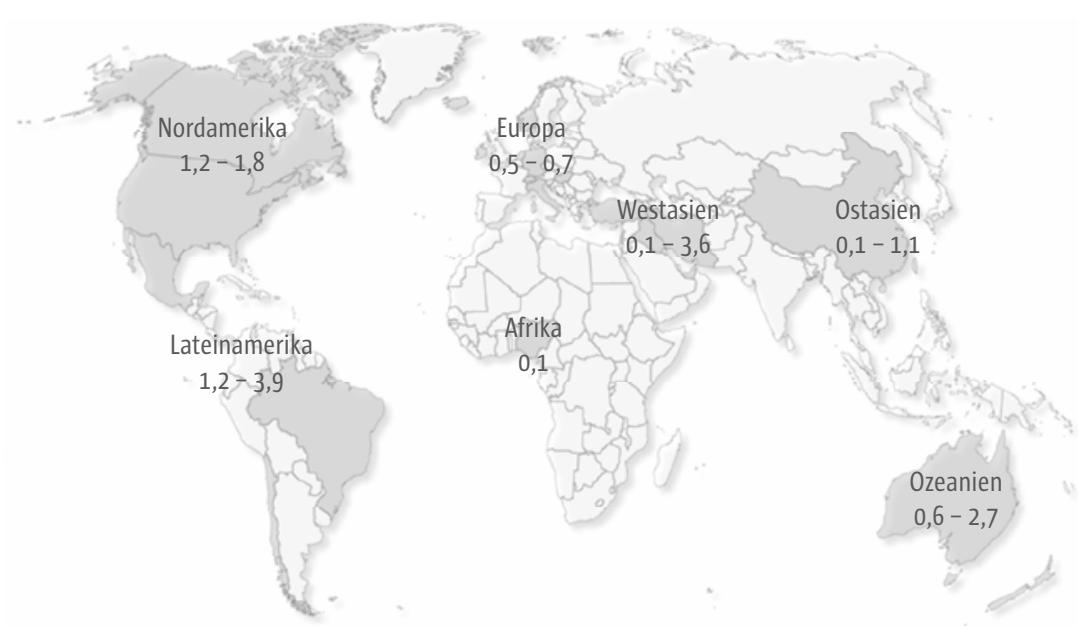

Abb. 41 Bereich der Jahresprävalenz (\%) von Zwangsstörungen in sieben Regionen der Welt

3. nicht-asiatische Länder: Jahresprävalenz o,8\% (95\% Konfidenzintervall: 0,5\%-1,3\%), Lebenszeitprävalenz 1,7\% (95\% Konfidenzintervall: $1,1 \%$ bis $2,4 \%$ )

Während nach Somers et al. (2006) asiatische Länder deutlich geringere Periodenprävalenzen für Zwangsstörungen aufweisen als nicht-asiatische Länder, zeigen aktuellere Studien jedoch, dass insbesondere in west- und südwestasiatischen Nationen, wie der Türkei und dem Irak, höhere Häufigkeitsraten erhoben wurden, als in den meisten anderen Nationen. Da jedoch aus den meisten Regionen, insbesondere aus asiatischen und afrikanischen Nationen, kaum Replikationsstudien vorliegen, sind internationale Vergleiche hinsichtlich der Häufigkeit von Zwangsstörungen derzeit nur bedingt aussagekräftig.

Zusammenfassend kann somit festgestellt werden, dass sich hinsichtlich der Häufigkeit von Zwangsstörungen im internationalen Vergleich einige regionale Unterschiede zeigen, wobei diese wohl vor allem methodologische Ursachen haben dürften. Ferner liegen zurzeit aus vielen Regionen keine Replikationsstudien vor, sodass bezüglich der erhobenen Prävalenzraten keine sicheren Aussagen über kulturell bedingte Unterschiede getroffen werden können. Basierend auf der gegenwärtigen Datenlage scheint es jedoch so zu sein, dass Zwangsstörungen im amerikanischen Nationen häufiger diagnostiziert werden als in Europa und dass diese in ost- und südostasiatischen Ländern seltener aufscheinen. West- und südwestasiatische Länder zeigen diesbezüglich derzeit widersprüchliche Ergebnisse.

\subsection{Kulturspezifische Zwangsphänomene}

Zwanghafte Verhaltensweisen im Zusammenhang mit sozialen Normen oder religiösen Riten können in zahlreichen Kulturkreisen beobachtet werden. Sie 
sind zumeist gut in den gesellschaftlichen Kontext eingebunden und werden daher toleriert und als Ausdruck einer ausgeprägten Gewissenhaftigkeit oder Religiosität geachtet. Die Übergänge zu individuellem Leiden und einer Beeinträchtigung der sozialen Funktionsfähigkeit sind dabei für kulturfremde Betrachter oftmals schwer zu ziehen, weshalb die pathologische Wertigkeit und Angemessenheit derartiger Phänomene am ehesten von Vertretern derselben Kultur beurteilt werden kann (Machleidt u. Calliess 2011). Kulturspezifische Zwangsstörungen stellen somit Verhaltens- und Erlebnisstörungen im Zuge kulturüblicher Rituale und Usancen dar, die auch kulturintern als abnorm angesehen werden (Friedmann 2005). Stehen Zwangsphänomene im Zusammenhang mit religiösen Vorschriften oder Ritualen, werden psychische Auffälligkeiten trotz des Leidensdrucks der Betroffenen oftmals weniger als psychologisches, sondern vielmehr als religiöses Problem angesehen.

Während „Ritual“ (lat. ritus: Brauchtum, Gewohnheit) ursprünglich „Gottesdienst“ oder die schriftlichen Anweisungen dazu bedeutete, bezieht sich der moderne Ritualbegriff auf symbolische Handlungen ganz allgemein, die in fast allen Bereichen des kulturellen Lebens zu finden sind. Ein Ritual kann als stilisiertes Verhalten definiert werden, das als besonderes Ereignis an einem besonderen Ort, $\mathrm{zu}$ einer besonderen Zeit und $\mathrm{zu}$ einem besonderen Anlass ausgeführt wird und dessen Grundfunktion es ist, persönliche und soziale Wirklichkeit zu konstruieren, indem das Zufällige und Unkontrollierbare des menschlichen Lebens durch Symbole geordnet wird. Rituelle Handlungen sind dabei stets in den jeweiligen kulturellen Kontext eingebunden und greifen auf vorgefertigte Handlungsabläufe zurück, sodass durch ihre regelhafte Ausführung die Zugehörigkeit zu einer Gemeinschaft ausgedrückt und verwirklicht wird. In der modernen Ritualtheorie werden ritualisierte Verhaltensweisen deshalb auch als kulturelle Performance verstanden, in der die Handlungsund Bedeutungsmuster einer Gesellschaft dargestellt und reproduziert werden (Belliger u. Krieger 2008).

In der klassischen Ritualtheorie wird den Reinheitsvorschriften hierbei ein besonderer Stellenwert zugesprochen, wobei es als eher unwichtig gilt, was in einer Kultur als „schmutzig“, „unrein“ oder ,abscheulich“ betrachtet wird. Wesentlich ist vielmehr, dass symbolische und moralische Grenzen gezogen werden, da solche binären Unterscheidungen, wie jene zwischen „gut und böse“ oder „rein und unrein“, der kulturellen Welt Struktur verleihen und dem Individuum als Verhaltensorientierung dienen (Belliger $\mathrm{u}$. Krieger 2008). In der regelmäßigen Wiederholung ritueller Handlungen wird dabei die angstbesetzte Umwelt stets aufs Neue geordnet und somit ein Gefühl von Sicherheit geschaffen.

Kulturen, in denen traditionelle Riten und religiöse Zeremonielle ein hohes Ansehen genießen, fordern zumeist ein strenges Einhalten von sittlichen Geboten, wobei ein Nichtbeachten zu einer Ausgrenzung aus dem Sozialverband führen kann. In diesen traditionsbestimmten Gemeinschaften wird zwang- 
haftes Verhalten, verstanden als ein Übermaß an Gewissenhaftigkeit und Pflichtbewusstsein, oft als Ausdruck einer tiefen Religiosität angesehen. Von kulturell sanktionierten Phänomenen muss jedoch die Zwangsstörung als psychische Erkrankung abgegrenzt werden, da sie bei den Betroffenen zu einer erheblichen Einschränkung des psychosozialen Funktionsniveaus sowie zu ausgeprägtem Leid führt. Ritualisierte Verhaltensweisen sind dabei nur dann als ein Hinweis auf eine psychische Störung anzusehen, wenn sie kulturelle Normen überschreiten und zu Zeiten oder an Orten auftreten, die von anderen Angehörigen der gleichen Kultur als unangemessen beurteilt werden (APA 200o). Überschreitet das rituelle Handeln einer Person jedoch die kulturellen Normen oder treten ritualisierte Verhaltensweisen zu Zeiten oder an Orten auf, die von anderen Angehörigen der gleichen Kultur als unangemessen beurteilt werden, kann dies Ausdruck einer Zwangsstörung sein (APA 200o).

Beobachtungen in Regionen mit vorwiegend muslimischer Bevölkerung zeigten, dass in islamischen Kulturen zwanghafte Vorstellungen und Verhaltensweisen oft eng mit religiösen Geboten und Riten verbunden sein können. So berichtete bereits Pfeiffer (1994), dass bei den strenggläubigen Muslimen auf Java ein umschriebenes Zwangssyndrom im Zusammenhang mit Reinigungsritualen bekannt ist, welches die Bezeichnung „Waswas“ trägt und die Bedeutung von „Zaudern“ und „Unsichersein“ hat (Pfeiffer 1994). Ein von diesem Syndrom Betroffener steigert dabei die dreimalige körperliche Waschung vor dem Gebet, das „Nehmen des Wassers“ (Wudhu), über das vorgeschriebene Maß hinaus, da er sich durch das anhaltende Gefühl der Unreinheit dazu gezwungen fühlt. Eine ähnliche Reaktion zeigt sich auch bei der Anrufung Gottes, die ebenfalls des Öfteren wiederholt werden muss, da aufdrängende Zweifel die Richtigkeit der Durchführung infrage stellen. Nachdem dieses religiöse Zwangsverhalten bereits im 11. Jahrhundert von dem persischen Theologen und Mystiker Abu Hamid Muhammad ibn Muhammad al-Ghazali (1058-1111) erwähnt wurde, weiß man heute, dass dieses Syndrom bei Gläubigen aller islamischen Länder und so auch bei muslimischen Migranten in Europa zu finden ist.

Die rituellen Vorschriften im Islam werden von praktizierenden Muslimen in der Regel nicht als belastend erlebt und so lässt sich aus der Verknüpfung verwandter anthropologischer Phänomene wie Regel, Ritual und Zwang auch verstehen, dass diese Verhaltensauffälligkeiten primär als religiöses und weniger als psychologisches Problem angesehen werden. Die Zwangsphänomene werden dabei als Anfechtung betrachtet, weil sie den Menschen von seinem Auftrag der Heiligung abhalten. Im Sinne eines kulturellen Krankheitskonzeptes gilt der Satan als Urheber dieses Syndroms, welcher den positiven Gedanken des Gläubigen durch Einflüsterung eine negative Gegenbewegung zu geben vermag. Während das Waswas-Syndrom im islamischen Kulturkreis vorwiegend als religiöse Eigenheit betrachtet wird, erleben die Betroffenen den Reinigungszwang als ich-dyston und als äußerst belastend. Die gesellschaftlich-religiöse Einordnung dieses Phänomens könnte nach Pfeiffer je- 
doch erklären, weshalb sich trotz des Leidensdrucks der Betroffenen nur selten eine Dekompensation ins sozial Trennende und Untragbare entwickelt (Pfeiffer 1994).

Ähnliche Zwangsphänomene im Zusammenhang mit religiösen Ritualen und Praktiken sind auch aus anderen Kulturkreisen wie dem Judentum und Hinduismus bekannt, wo ein übermäßiges Rezitieren von Mantras, zwanghaftes Baden oder ein übergewissenhaftes Einhalten von Speisevorschriften beschrieben wurden (Friedmann 2005). Nach Greenberg und Huppert (2010) beträgt die Häufigkeit religiöser Symptome bei Zwangspatienten zwischen o\% und 93\%, wobei die Prävalenzraten in traditionellen islamischen Ländern sowie im Mittleren Osten grundsätzlich höher sind als in westlichen, indischen oder südostasiatischen Nationen (Greenberg u. Huppert 2010; Tek u. Ulug 2001) (s. Tab. 19).

\section{Tab. 19 Häufigkeit religiöser Symptome (\%) bei Zwangsstörungen im internationalen Vergleich (modifiziert nach Greenberg u. Huppert 2010)}

\begin{tabular}{|l|c|}
\hline Nation & religiöse Symptome (\%) \\
\hline Großbritannien & $0-5$ \\
\hline USA & $10-38$ \\
\hline Indien & $4-31$ \\
\hline Japan & 8 \\
\hline Türkei & $11-42$ \\
\hline Ägypten & 60 \\
\hline Saudi Arabien & $>50$ \\
\hline Bahrain & 40 \\
\hline Israel & $41-93$ \\
\hline
\end{tabular}

In zahlreichen Studien konnte diesbezüglich nachgewiesen werden, dass weder die religiöse Zugehörigkeit noch die persönliche Religiosität die Entstehung einer Zwangsstörung beeinflussen (Greenberg $u$. Huppert 2010; Tek $u$. Ulug 2001). Ist jedoch bei einem Zwangskranken der soziokulturelle Hintergrund religiös und traditionell geprägt und besitzen religiöse Ansichten einen idiosynkratisch bedeutsamen Stellenwert, dann ist es wahrscheinlich, dass der Betroffene religiöse Symptome entwickeln wird. Denn der Inhalt der Zwangssymptome spiegelt allgemeine besorgniserregende als auch persönlich relevante Themen wider, ob diese nun das Werk des Teufels, die Verunreinigung durch Keime oder das Risiko an einer erworbenen Immunschwäche zu erkranken betreffen.

In Anlehnung an das kognitive Modell der Zwangsstörung (Salikovskis 1985), nach welchem normale intrusive Gedanken durch eine dysfunktionale Bewer- 


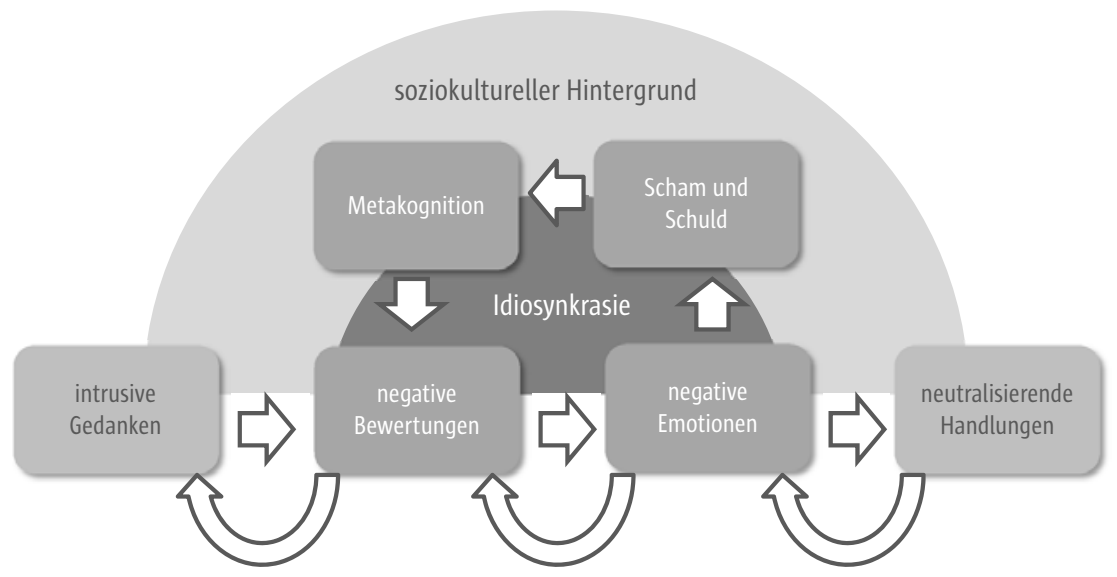

Abb. 42 Soziokulturelle und idiosynkratische Bereiche im kognitiven Modell der Zwangsstörung (modifiziert nach Salikovskis 1985)

tung obsessiven Charakter erhalten und so negative Emotionen evozieren, welche schließlich zu neutralisierenden Handlungen (Kompulsionen) drängen, kann der Einfluss der Kultur auf die Symptomatik von Zwangsphänomenen nicht auf den Inhalt, sondern auch im Bereich der Metakognitionen sowie der sozialorientierten Gefühle angenommen werden (s. Abb. 42). So prägt der soziokulturelle Hintergrund wohl nicht nur den Inhalt von obsessiven Gedanken und kompulsiven Handlungen, sondern färbt auch das idiosynkratische Erleben des psychischen Zwangs, was letztlich Einfluss auf regionale Häufigkeitsraten der Zwangsstörung haben könnte. Leider fehlen gegenwärtig entsprechende kulturvergleichende Untersuchungen, welche auch jene Aspekte ausreichend mitbeachten.

\section{Zusammenfassung}

Die Zwangsstörung ist eine schwerwiegende psychische Erkrankung mit zumeist chronischem Verlauf, die von einem massiven Leidensdruck und einer ausgeprägten Beeinträchtigung der Lebensqualität begleitet wird. Das wesentliche Kennzeichen dieser Störung sind wiederkehrende Vorstellungen und stereotype Verhaltensweisen, welche von den Betroffenen als quälend empfunden werden. Da der Inhalt von Zwangserscheinungen oftmals sehr persönlich ist oder gar konträr zum soziokulturellen Wertesystem steht, werden diese in der Regel von ausgeprägten Scham- und Schuldgefühlen begleitet. So besteht bei Zwangskranken auch eine deutliche Neigung die Beschwerden vor dem Umfeld zu verheimlichen und die Fassade einer normalen Lebensführung aufrecht zu erhalten (Vorderholzer et al. 2011). Aufgrund dieser Verheimlichungstendenz der Betroffenen wurde auch die Häufigkeit der Zwangserkrankung in der Allgemeinbevölkerung über lange Zeit unterschätzt. Heute weiß man, dass es sich bei der Zwangsstörung um ein weltweit anzutreffendes Krankheitsbild mit weitgehend einheitlichen Prävalenzraten handelt, weshalb die formale Dimension der Störung als vorwiegend biologisch begründet angesehen wird. Dennoch finden sich deutliche Unterschiede im Bereich der inhaltlichen Ausgestaltung der Symptomatik. Kultur beein- 
flusst die Gestaltung von Zwangsphänomenen dabei durch internalisierte Symbolsysteme, wobei Leitbilder und Ängste einer Gemeinschaft in Form einer epochalen Bedingtheit (Pfeiffer 1994) zum Ausdruck zu kommen.

\section{Literatur}

Abou-Saleh MT, Ghubash R, Daradkeh TK (2001) Al Ain Community Psychiatric Survey I. Prevalence and sociodemographic correlates. Soc Psychiatry Psychiatr Epidemiol 36(1), 20-28

Adam Y, Meinlschmidt G, Gloster AT, Lieb R (2012) Obsessive-compulsive disorder in the community: 12-month prevalence, comorbidity and impairment. Soc Psychiatry Psychiatr Epidemiol 47(3), 339-349

Akhtar S, Wig NN, Varma VK, Pershad D, Verma SK (1975) A phenomenological analysis of symptoms in obsessivecompulsive neurosis. Br | Psychiatry 127, 342-348

Alhasnawi S, Sadik S, Rasheed M, Baban A, Al-Alak MM, Othman AY, Othman Y, Ismet N, Shawani 0, Murthy S, Aljadiry M, Chatterji S, Al-Gasseer N, Streel E, Naidoo N, Mahomoud Ali M, Gruber M), Petukhova M, Sampson NA, Kessler RC (2009) Iraq Mental Health Survey Study Group. The prevalence and correlates of DSM-IV disorders in the Iraq Mental Health Survey (IMHS). World Psychiatry 8(2), 97-109

American Psychiatric Association (2010) Diagnostic and Statistical Manual of Mental Disorders, Fourth Edition, Text Revision. American Psychiatric Association Washington DC

American Psychiatric Association (2013) Diagnostic and Statistical Manual of Mental Disorders, Fifth Edition. American Psychiatric Association Arlington VA

Andrade L, Walters EE, Gentil V, Laurenti R (2002) Prevalence of ICD-10 mental disorders in a catchment area in the city of São Paulo, Brazil. Soc Psychiatry Psychiatr Epidemiol 37(7), 316-325

Andrade LH, Wang YP, Andreoni S, Silveira CM, Alexandrino-Silva C, Siu ER, Nishimura R, Anthony IC, Gattaz WF, Kessler RC, Viana MC (2012) Mental disorders in megacities: findings from the São Paulo Megacity Mental Health Survey, Brazil. PLoS One 7(2):e31879. doi: 10.1371/journal.pone.0031879

Andrews G, Henderson S, Hall W (2001) Prevalence, comorbidity, disability and service utilisation. Overview of the Australian National Mental Health Survey. Br | Psychiatry 178, 145-153

Angst |, Gamma A, Endrass |, Goodwin R, Ajdacic V, Eich D, Rössler W (2004) Obsessive-compulsive severity spectrum in the community: prevalence, comorbidity, and course. Eur Arch Psychiatry Clin Neurosci. 254(3), 156-164

Belliger A, Krieger DI (Hrsg.) (2008) Ritualtheorien. Ein einführendes Handbuch. Verlag für Sozialwissenschaften Wiesbaden

Berrios GE (1989) Obsessive-compulsive disorder: its conceptual history in France during the 19th century. Compr Psychiatry 30(4), 283-295

Berrios GE (1996) The history of mental symptoms. Descriptive psychopathology since the nineteenth century. Cambridge University Press New York

Bieri P (2011) Das Handwerk der Freiheit. Über die Entdeckung des eigenen Willens (Erstauflage 2001). 10. Auflage. Fischer Taschenbuch Verlag Frankfurt am Main

Bijl RV, Ravelli A, van Zessen G (1998) Prevalence of psychiatric disorder in the general population: results of The Netherlands Mental Health Survey and Incidence Study (NEMESIS). Soc Psychiatry Psychiatr Epidemiol 33(12), 587-595

Binder H (1979) Zur Psychologie der Zwangsvorgänge (1936). In: Ausgewählte Arbeiten. Band 1. 221-317. Huber Verlag Bern

Bland RC, Newman SC, Orn H (1987) Period prevalence of psychiatric disorders in Edmonton. Acta Psychiatr Scand 77 (suppl. 338), 33-42

Bland RC, Orn H, Newman SC (1988) Lifetime prevalence of psychiatric disorders in Edmonton. Acta Psychiatr Scand 77(suppl. 338), 24-32

Bloch MH, Landeros-Weisenberger A, Rosario MC, Pittenger C, Leckman JF (2008) Meta-analysis of the symptom structure of obsessive-compulsive disorder. Am | Psychiatry 165(12), 1532-1542

Bunting BP, Murphy SD, O'Neill SM, Ferry FR (2012) Lifetime prevalence of mental health disorders and delay in treatment following initial onset: evidence from the Northern Ireland Study of Health and Stress. Psychol Med 42(8), 1727-1739 
Bürgy M (2005) Zwangsstörung und Schizophrenie. Versuch einer psychopathologischen Differenzierung am Einzelphänomen Zwang. Nervenarzt 76(11), 1370-1375

Canino G), Bird HR, Shrout PE, Rubio-Stipec M, Bravo M, Martinez R, Sesman M, Guevara LM (1987) The prevalence of specific psychiatric disorders in Puerto Rico. Arch Gen Psychiatry 44(8), 727-735

Caraveo-Anduaga II, Bermúdez EC (2004) The epidemiology of obsessive-compulsive disorder in Mexico City. Salud Mental 27(2)

Chen CN, Wong I, Lee N, Chan-Ho MW, Lau JT, Fung M (1993) The Shatin Community Mental Health Survey in Hong Kong: II major findings. Arch Gen Psychiatry 50(2), 125-133

Cho MJ, Kim JK, Jeon HJ, Suh T, Chung IW, Hong IP, Bae JN, Lee DW, Park JI, Cho SI, Lee CK, Hahm BI (2007) Lifetime and 12-month prevalence of DSM-IV psychiatric disorders among Korean adults. I Nerv Ment Dis 195(3), 203-10

Cilli AS, Telcioglu M, Așkin R, Kaya N, Bodur S, Kucur R (2004) Twelve-month prevalence of obsessive-compulsive disorder in Konya, Turkey. Compr Psychiatry 45(5), 367-374

Crino R, Slade T, Andrews $G(2005)$ The changing prevalence and severity of obsessive-compulsive disorder criteria from DSM-III to DSM-IV. Am I Psychiatry 162(5), 876-882

Degonda M, Wyss M, Angst I (1993) The Zurich Study. XVIII. Obsessive-compulsive disorders and syndromes in the general population. Eur Arch Psychiatry Clin Neurosci 243(1), 16-22

Dilling H, Mombour W, Schmidt MH, Schulte-Markwort E (Hrsg.) (2006) Weltgesundheitsorganisation. Internationale Klassifikation psychischer Störungen. ICD-10 Kapital V(F). Diagnostische Kriterien für Forschung und Praxis. 4. Auflage. Verlag Hans Huber Bern

Donath I (1896) Zur Kenntnis des Anancasmus (psychische Zwangszustände). Archiv für Psychiatrie und Nervenkrankheiten 29(1), 211-224

Eaton WW, Regier DA, Locke BZ, Taube CA (1981) The Epidemiologic Catchment Area Program of the National Institute of Mental Health. Public Health Rep 96(4), 319-325

Esquirol E (1839) Des maladies mentales considérées sous les rapports médical, hygiénique et médico-legal. Baillière Paris

Faravelli C, Guerrini Degl'Innocenti B, Biardinelli L (1989) Epidemiology of anxiety disorders in Florence. Acta Psychiatr Scand 79(4), 308-312

Florescu S, Ciutan M, Popovici G, Galaon M, Ladea M, Pethukova M, Hoffnagle A (2009) The Roman Mental Health Study: main aspects of lifetime prevalence and service use of DSM-IV disorders. Management in Health 3, 22-30

Foa EB, Kozak M], Goodman WK, Hollander E, Jenike MA, Rasmussen SA (1995) DSM-IV field trial: obsessivecompulsive disorder. Am / Psychiatry 152(1), 90-96

Friedmann A (2005) Psychiatrie und Menschenbild. Wien Med Wochenschr 155 (23-24), 517-523

Fullana MA, Vilagut G, Rojas-Farreras S, Mataix-Cols D, de Graaf R, Demyttenaere K, Haro JM, de Girolamo G, Lépine IP, Matschinger H, Alonso I and for the ESEMeD/MHEDEA 2000 investigators (2010) Obsessivecompulsive symptom dimensions in the general population: results from an epidemiological study in six European countries. I Affect Disord 124(3), 291-299

Gebsattel VE (1983) Die Welt des Zwangskranken. Mschr f Psychiatrie u Neurologie 99, 10-74

Grabe HJ, Meyer C, Hapke U, Rumpf HJ, Freyberger HJ, Dilling H, John U (2000) Prevalence, quality of life and psychosocial function in obsessive-compulsive disorder and subclinical obsessive-compulsive disorder in northern Germany. Eur Arch Psychiatry Clin Neurosci 250(5), 262-268

Grabe HJ, Meyer C, Hapke U, Rumpf HJ, Freyberger HJ, Dilling H, John U (2001) Lifetime-comorbidity of obsessive-compulsive disorder and subclinical obsessive-compulsive disorder in northern Germany. Eur Arch Psychiatry Clin Neurosci 251(3), 130-135

Greenberg D, Huppert JD (2010) Scrupulosity: A unique subtype of obsessive-compulsive disorder. Curr Psychiatry Rep 12(4), 282-289

Griesinger W (1868) Über einen wenig bekannten psychopathischen Zustand. Vortrag gehalten in der Berliner Medicinisch-Psychologische Gesellschaft (Sitzungsprotokoll vom 23. März 1868). Archiv für Psychiatrie und Nervenkrankheiten 1(3), 626-635

Gureje 0, Lasebikan V0, Kola L, Makanjuola VA (2006) Lifetime and 12-month prevalence of mental disorders in the Nigerian Survey of Mental Health and Well-Being. Br | Psychiatry 188, 465-471 
Hwu HG, Yeh EK, Chang LY (1989) Prevalence of psychiatric disorders in Taiwan defined by the Chinese Diagnostic Interview Schedule. Acta Psychiatr Scand 79(2), 136-147

Jacobi F, Wittchen HU, Holting C, Höfler M, Pfister H, Müller N, Lieb R (2004) Prevalence, co-morbidity and correlates of mental disorders in the general population: results from the German Health Interview and Examination Survey (GHS). Psychol Med 34(4), 597-611

Jaspers K (1973) Allgemeine Psychopathologie. 9., unveränderte Auflage. Springer Verlag Berlin Heidelberg New York

Kapfhammer HP (2008) Zwangsstörungen. In: Möller HJ, Laux G, Kapfhammer HP (Hrsg.) Psychiatrie und Psychotherapie. 1635-1660. Springer Verlag Berlin Heidelberg

Karam EG, Mneimneh ZN, Karam AN, Fayyad JA, Nasser SC, Chatterji S, Kessler RC (2006) Prevalence and treatment of mental disorders in Lebanon: a national epidemiological survey. Lancet 367(9515), 1000-1006

Karno M, Golding JM, Sorenson SB, Burnam MA (1988) The epidemiology of obsessive-compulsive disorder in five US communities. Arch Gen Psychiatry 45(12), 1094-1099

Kessler RC, Berglund P, Demler 0, Jin R, Merikangas KR, Walters EE (2005) Lifetime prevalence and age-of-onset distributions of DSM-IV disorders in the National Comorbidity Survey Replication. Arch Gen Psychiatry 62(6), 593-602

Kessler RC, Chiu WT, Demler 0, Walters EE (2005) Prevalence, severity and comorbidity of 12-month DSM-IV disorders in the National Comorbidity Survey Replication. Arch Gen Psychiatry 62(6), 617-627

Kohl F (1998) Die Dimension der (inversen) „Territorialität“ bei Zwangsstörungen. Ethologische Aspekte, aufgezeigt an einer Kasuistik. Psychotherapie 3(2), 258-262

Kringlen E, Torgersen S, Cramer V (2001) A Norwegian Psychiatric Epidemiological Study. Am I Psychiatry 158(7), 1091-1098

Leckman JF, Denys D, Simpson HB, Mataix-Cols D, Hollander E, Saxena S, Miguel EC, Rauch SL, Goodman WK, Phillips KA, Stein DI (2010) Obsessive-compulsive disorder: A review of the diagnostic criteria and possible subtypes and dimensional specifiers for DSM-V. Depress Anxiety 27(6), 507-527

Leckman JF, Griece DE, Boardman I, Zhang H, Vitale A, Bondi C, Alsobrook I, Peterson BS, Cohen DI, Rasmussen SA, Goodman WK, McDougle Cl, Pauls DL (1997) Symptoms of obsessive-compulsive disorder. Am I Psychiatry 154(7), 911-917

Lee CK, Kwak YS, Rhee H, Kim YS, Han JH, Choi J0, Lee YH (1987) The nationwide epidemiological study of mental disorders in Korea. I Korean Med Sci 2(1), 19-34

Loewenfeld L (2007) Die psychischen Zwangserscheinungen. Auf klinischer Grundlage dargestellt. Edition Classic. VDM Verlag Dr. Müller Saarbrücken

Machleidt W, Calliess T (2011) Transkulturelle Aspekte psychischer Erkrankungen. In: Möller HJ, Laux G, Kampfhammer HP (Hrsg.) Psychiatrie, Psychosomatik, Psychotherapie. Springer Verlag, Berlin Heidelberg

Mataix-Cols D, Rosario-Campos MC, Leckman JF (2005) A multidimensional model of obsessive-compulsive disorder. Am I Psychiatry 162(2), 228-238

McEvoy PM, Grove R, Slade T (2011) Epidemiology of anxiety disorders in the Australian general population: findings of the 2007 Australian National Survey of Mental Health and Wellbeing. Aust NZ) Psychiatry 45(11), 957-967

Mohammadi MR, Ghanizadeh A, Rahgozar M, Noorbala AA, Davidian H, Afzali HM, Naghavi HR, Yazdi SA, Saberi SM, Mesgarpour B, Akhondzadeh S, Alaghebandrad I, Tehranidoost M (2004) Prevalence of obsessivecompulsive disorder in Iran. BMC Psychiatry 14(4)

Morschitzky H (2009) Angststörungen. Diagnostik, Konzepte, Therapie, Selbsthilfe. 4. Auflage. Springer Verlag Wien

Németh A, Szádóczky E, Treuer T, Vandalik E, Papp Z (1997) (P.3.027) Epidemiology of OCD in Hungary. Eur Neuropsychopharmacol 7 (suppl. 2), 234

Oakley Browne MA, Wells JE, Scott KM, McGee MA (2006) New Zealand Mental Health Survey Research Team. Lifetime prevalence and projected lifetime risk of DSM-IV disorders in Te Rau Hinengaro: the New Zealand Mental Health Survey. Aust NZ) Psychiatry 40(10), 865-874

Oakley-Browne MA, Joyce PR, Wells JE, Bushnell JA, Hornblow AR (1989) Christchurch Psychiatric Epidemiology Study, Part II: Six month and other period prevalences of specific psychiatric disorders. Aust NZ) Psychiatry 23(3), 327-340 
Pfeiffer WM (1994) Transkulturelle Psychiatrie. Ergebnisse und Probleme. Thieme Verlag Stuttgart

Regier DA, Myers JK, Kramer M, Robins LN, Blazer DG, Hough RL, Eaton WW, Locke BZ (1984) The NIMH Epidemiologic Catchment Area program. Historical context, major objectives, and study population characteristics. Arch Gen Psychiatry 41(10), 934-941

Reynolds EH, Kinnier Wilson JV (2012) Obsessive compulsive disorder and psychopathic behaviour in Babylon. I Neurol Neurosurg Psychiatry 83(2), 99-201

Ruscio AM, Stein DI, Chiu WT, Kessler RC (2010) The epidemiology of obsessive-compulsive disorder in the National Comorbidity Survey Replication. Mol Psychiatry 15(1), 53-63

Salkovskis PM (1985) Obsessional-compulsive problems: a cognitive-behavioural analysis. Behav Res Therapy 25, 571-583

Scharfetter C (2002) Allgemeine Psychopathologie. Eine Einführung. 5. Auflage. Georg Thieme Verlag Stuttgart Schneider K (1925) Zwangszustände und Schizophrenie. Archiv für Psychiatrie und Nervenkrankheiten 74(1), 93-107 Schneider K (2007) Klinische Psychopathologie. 15. Auflage. Georg Thieme Verlag Stuttgart New York

Somers JM, Goldner EM, Waraich P, Hsu L (2006) Prevalence and incidence studies of anxiety disorders: a systematic review of the literature. Can I Psychiatry 51(2), 100-113

Stefansson JG, Lindal E, Björnsson JK, Guomundsdottir A (1991) Lifetime prevalence of specific mental disorders among people born in Iceland in 1931. Acta Psychiatr Scand 84(2), 142-149

Stompe T (2011) Schizophrenie und Zwang. Jatros Neurologie u. Psychiatrie 9(4), 30-34

Subramaniam M, Abdin E, Vaingankar JA, Chong SA (2012) Obsessive-compulsive disorder: prevalence, correlates, help-seeking and quality of life in a multiracial Asian population. Soc Psychiatr Epidemiol 47(12), 2035-2043

Süllwold L (2006) Veränderungen am tradierten Konzept der Zwangsstörung. In: Schneider F (Hrsg.) Entwicklungen der Psychiatrie. Springer Verlag Berlin-Heidelberg New York

Tek C, Ulug B (2001) Religiosity and religious obsessions in obsessive-compulsive disorder. Psychiatry Res 104(2), 99-108

Thomsen R (1895) Klinische Beiträge zur Lehre von den Zwangsvorstellungen und verwandten psychischen Zuständen. Archiv für Psychiatrie und Nervenkrankheiten 27(2), 319-385

Viana MC, Andrade LH (2012) Lifetime prevalence, age and gender distribution and age-of-onset of psychiatric disorders in the São Paulo metropolitan area, Brazil: results from the São Paulo Megacity Mental Health Survey. Rev Bras Psiquiatr 34, 249-260

Vicente B, Kohn R, Rioseco P, Saldivia S, Levav I, Torres S (2006) Lifetime and 12-month prevalence of DSM-III-R disorders in the Chile psychiatric prevalence study. Am I Psychiatry 163(8), 1362-1370

Vorderholzer U, Schlegl S, Külz AK (2011) Epidemiologie und Versorgungssituation von Zwangsstörungen. Nervenarzt 82(3), 273-280

Weissman MM, Bland RC, Canino GJ, Greenwald S, Hwu HG, Lee CK, Newman SC, Oakley-Browne MA, Rubio-Stipec M, Wickramaratne PJ, Wittchen HU, Yeh EK (1994) The cross national epidemiology of obsessive compulsive disorder. I Clin Psychiatry 55(3), 5-10

Wells JE, Browne MA, Scott KM, McGee MA, Baxter |, Kokaua I (2006) Prevalence, interference with life and severity of 12 month DSM-IV disorders in Te Rau Hindegaro: the New Zealand Mental Health Survey. Aust NZJ Psychiatry 40(10), 845-854

Wells JE, Bushnell JA, Hornblow AR, Joyce PR, Oakley-Browne MA (1989) Christchurch Psychiatric Epidemiology Study, Part I: Methodology and lifetime prevalence for specific psychiatric disorders. Aust NZ) Psychiatry 23(3), $315-326$

Westphal C (1878) Über Zwangsvorstellungen. Berliner Medicinisch-Psychologische Gesellschaft, Sitzungsprotokolle 5. März 1877 und 16. April 1877. Archiv für Psychiatrie und Nervenkrankheiten 8(3), 734-764

Wille L (1882) Zur Lehre von den Zwangsvorstellungen. Archiv für Psychiatrie und Nervenkrankheiten 12(1), 1-43 Wittchen HU, Jacobi F (2005) Size and burden of mental disorders in Europe - a critical review and appraisal of 27 studies. Eur Neuropsychopharmacol 15(4), 357-376

Wittchen HU, Jacobi F, Rehm I, Gustavsson A, Svensson M, Jönsson B, Olesen I, Allgulander C, Alonso |, Faravelli C, Fratiglioni L, Jennum P, Lieb R, Maercker A, van Os I, Preisig M, Salvador-Carulla L, Simon R, Steinhausen HC (2011) The size and burden of mental disorders and other disorders of the brain in Europe 2010. Eur Neuropschopharmacol 21(9) 655-679 


\subsection{Kulturspezifische Zwangsphänomene}

Zaudig M (2011a) Die Zwangsstörung. Krankheitsbild, Diagnostik und Therapie. Z Psychosom Med Psychother 57, 3-50

Zaudig M (2011b) Heterogenität und Komorbidität der Zwangsstörung. Nervenarzt 82, 290-298 